$16^{\text {th }}$ AIAA/CEAS Aeroacoustics Conference, Stockholm, Sweden, June 7-9, 2010 Jet Aeroacoustics Session AIAA-2010-3780

\title{
DAMAS Processing for a Phased Array Study in the NASA Langley Jet Noise Laboratory
}

\author{
Thomas F. Brooks* and William M. Humphreys, Jr. ${ }^{+}$ \\ NASA Langley Research Center, Hampton, Virginia 23681 \\ Gerald E. Plassman** \\ National Institute of Aerospace, Hampton, Virginia 23666
}

\begin{abstract}
A jet noise measurement study was conducted using a phased microphone array system for a range of jet nozzle configurations and flow conditions. The test effort included convergent and convergent/divergent single flow nozzles, as well as conventional and chevron dual-flow core and fan configurations. Cold jets were tested with and without wind tunnel co-flow, whereas, hot jets were tested only with co-flow. The intent of the measurement effort was to allow evaluation of new phased array technologies for their ability to separate and quantify distributions of jet noise sources. In the present paper, the array post-processing method focused upon is DAMAS (Deconvolution Approach for the Mapping of Acoustic Sources) for the quantitative determination of spatial distributions of noise sources. Jet noise is highly complex with stationary and convecting noise sources, convecting flows that are the sources themselves, and shock-related and screech noise for supersonic flow. The analysis presented in this paper addresses some processing details with DAMAS, for the array positioned at $90^{\circ}$ (normal) to the jet. The paper demonstrates the applicability of DAMAS and how it indicates when strong coherence is present. Also, a new approach to calibrating the array focus and position is introduced and demonstrated.
\end{abstract}

\section{INTRODUCTION}

The use of phased microphone arrays for the study of jet noise has expanded in recent years due to the promise of more in-depth understanding and quantification. Tester and Glegg ${ }^{1}$ reviewed array diagnostic methods for engine noise sources and found great importance in the emergence of array-beamforming deconvolution methods, particularly those able to determine coherence. Papamoschou and Dadar ${ }^{2}$ performed one-dimensional beamforming along a model jet axis using microphones mounted along a circular arc. A deconvolution method, based in part on that of DAMAS of Brooks and Humphreys ${ }^{3}$, was used to extract the axial source distribution from the array microphones' cross- and auto-spectra. They found that the deconvolution approach effectively sharpened the array resolution by a factor of at least five.

A more expansive jet-noise array study is reported by Dougherty and Podboy ${ }^{4}$. A planar array was used in the Small Hot Jet Acoustic Rig (SHJAR) in the Aero-Acoustic Propulsion Laboratory at the NASA-Glenn Research Center. Several 2-inch nozzles were tested over a range of sonic and subsonic, hot and cold, jet conditions. The study produced rather detailed mappings of source regions, including shock cell structures. Besides standard array beamforming of the jet noise region, an algorithm called TIDY was used to deconvolve the beamformed solutions. TIDY (not fully documented at the time of Ref.4) is stated to be similar philosophically to CLEAN-SC ${ }^{5}$, but

\footnotetext{
* Senior Research Scientist, AIAA Fellow

† Senior Research Scientist, AIAA Senior Member

** Senior Research Scientist, AIAA Member
} 
operates in the time, rather than frequency, domain. The algorithms iteratively remove (by decomposition) side lobes and parts of the beamform solutions that are spatially coherent with the apparent peak sources. The disposition and character of such spatial coherence within the noise regions are not defined by the method - but the sum (integration) of the TIDY noise-source regions were found in the study to be within several $\mathrm{dB}$ of the microphone auto-spectrum levels, indicating most energy is captured. In TIDY, the rather standard practice of using the Point Spread Function (PSD) as a basis for deconvolution, as is done in DAMAS ${ }^{3}$, DAMAS$\mathrm{C}^{8}, \mathrm{CLEAN}^{6}$, and DAMAS2 ${ }^{7}$, is avoided.

The study at NASA Langley Research Center was developed to examine the application of advanced phased array methodologies to the jet noise problem. Methodologies emphasized are DAMAS ${ }^{3}$, where deconvolution solutions can be couched in terms of distributions of uncorrelated sources, and DAMAS- $\mathrm{C}^{8}$, where the solutions are able to separate and define coherence between sources in the distributions. A range of single and dual stream jet nozzle configurations, sizes, temperatures, and simulated engine cycle conditions were tested. The jet engine simulator was operated with and without wind tunnel co-flow. A specially designed array, called JEDA (JEt Directional Array) was positioned at $90^{\circ}$ (normal) to the jet axis, and downstream of the jet at $115^{\circ}, 135^{\circ}$, and $150^{\circ}$. The present paper presents processed results using DAMAS for a range of jet noise test configurations with the array positioned normal to the jet axis. Introduced is a calibration conditioning method designed to correct steering-vector phase errors between microphones.

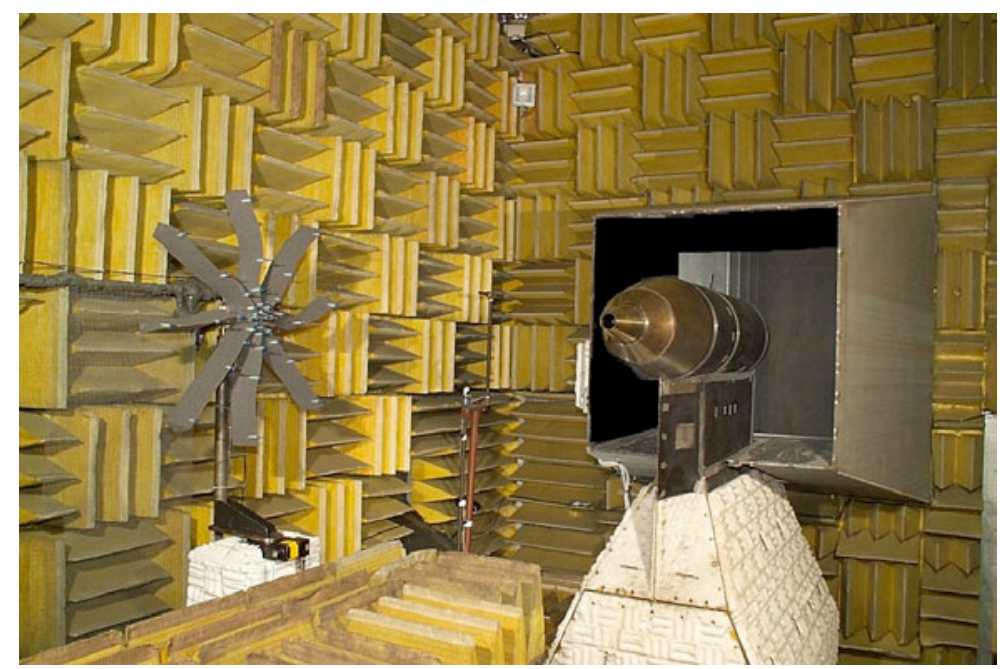

Figure 1. JEDA array is positioned in the LSAWT at the $90^{\circ}$ jet observation angle with the JES test apparatus.

\section{EXPERIMENTIAL PROGRAM}

Test Facility and Models. The test was performed in the anechoic Low Speed Aeroacoustics Wind Tunnel (LSAWT) of the Jet Noise Laboratory at NASA Langley Research Center. The various propulsive jet nozzles that were used were installed on the Jet Engine Simulator (JES), itself centered in an open-jet wind tunnel (WT) flow from a 4.7 by $4.7 \mathrm{ft}$ nozzle. The WT has a drawdown flow induced by the facility exhaust fan. The jet models are supplied with high-pressure air whose temperature is controlled by electric heaters and supplemented by propane burners as needed to simulate desired single stream nozzle flow and dual stream flow to match turbofan engine cycles. Figure 1 is a photograph of the installed microphone array system in the tunnel with a single stream 2.67" diameter convergent nozzle mounted on the JES. The chamber is fully covered with acoustic wedges during testing.

Both single and dual stream nozzles were tested. Nozzle lines are shown in Fig. 2, along with facility-assigned configuration numbers. The single stream configurations included a $\mathrm{D}=2.67$ " exit diameter convergent $(\mathrm{CV})$ nozzle and a convergent-divergent (CD) nozzle with a 2.67" throat diameter and D $=2.89$ " exit diameter. As shown in Figs. 2(a) and (b), both have internal plugs. The nozzle exits were placed $\mathrm{X}=63.2$ " and 64.1" downstream of the WT nozzle exit plane, respectively. More details are given by Norum ${ }^{9}$. Several dual flow configurations were tested. Fig. 2(c) shows a fan nozzle with a diameter of $\mathrm{D}=9.45$ " and a core convergent nozzle with $\mathrm{D}=2.86$ ". The respective exit downstream distances are $\mathrm{X}=55.75$ " and 63.2". It also has an internal-plug.

A series of chevron dual-stream nozzle configurations are shown in Figs. 2(d), (e), (f), and (g). These were comprised of round, eight chevron core nozzles, and sixteen-chevron fan nozzles. Shown are (d) baseline round nozzles, (e) round fan and chevron core nozzles, (f) chevron fan and round core nozzles, and (g) chevron fan and chevron core nozzles. For these, the fan nozzle $\mathrm{D}=9.45$ " at $\mathrm{X}=55.63$ ", and $(\mathrm{g})$ the core nozzle $\mathrm{D}=5.08$ " at $\mathrm{X}=$ 
59.88". These D and X dimensions are measured from the nozzle lip line or the midpoint of the chevron for the chevron nozzle cases. It is seen that these all have an external plug. The chevron core nozzles were aligned such that the horizontal centerline crossed the chevron troughs and the corresponding fan chevron tips, as shown in Fig. 3. Thomas and Kinzie ${ }^{10}$ report acoustic tests of these chevron nozzles (including with and without Pylon effects).

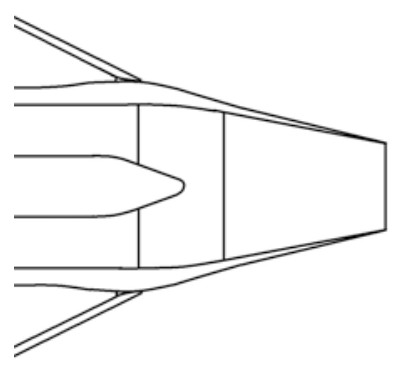

(a) Single CV; Conf. 30000

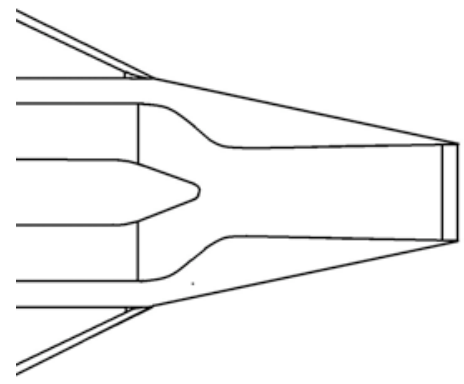

(b) Mach. 1.5, Single CD; Conf. 20000

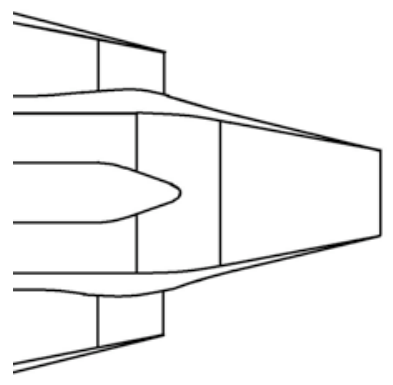

(c) BPR-11; Conf. 11000

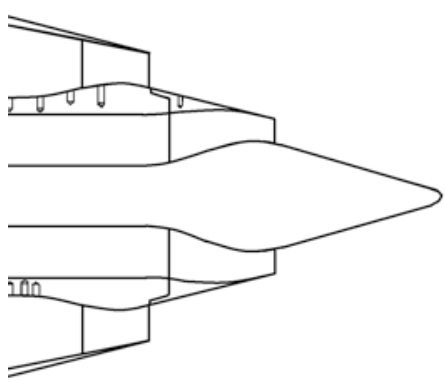

(d) BPR-5, Baseline Round; Conf. 50000

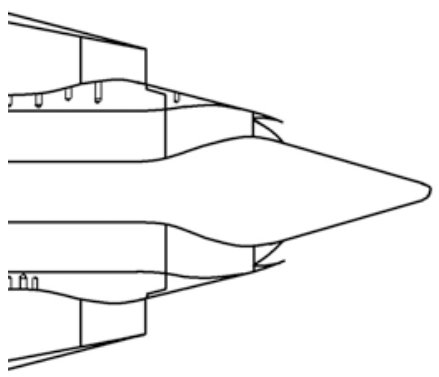

(e) BPR-5, Round Fan, Chevron Core; Conf. 50800

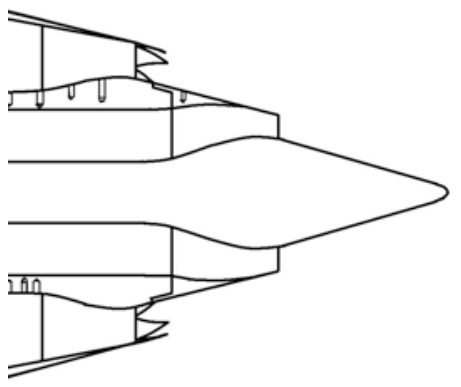

(f) BPR-5, Chevron Fan, Round Core; Conf. 50700

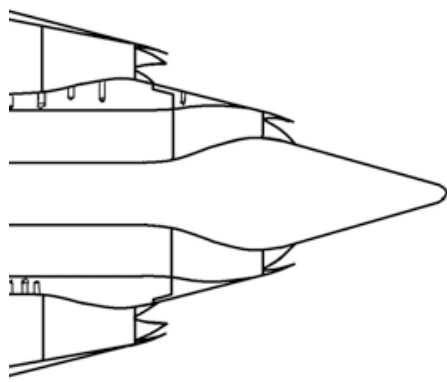

(g) BPR-5, Chevron Fan, Chevron Core; Conf. 50900

Figure 2. Configurations of nozzles used in the test. 


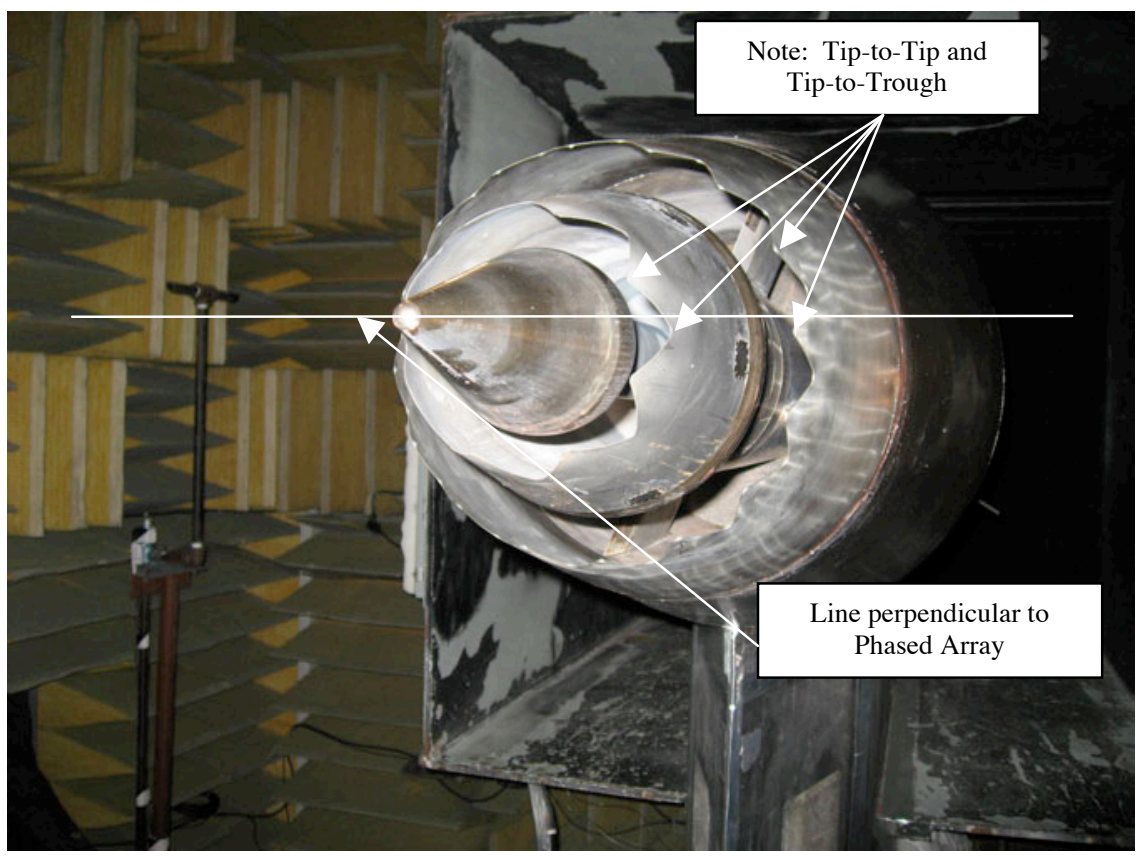

Figure 3. Orientations of chevron nozzles with respect to JEDA, Conf. 50900

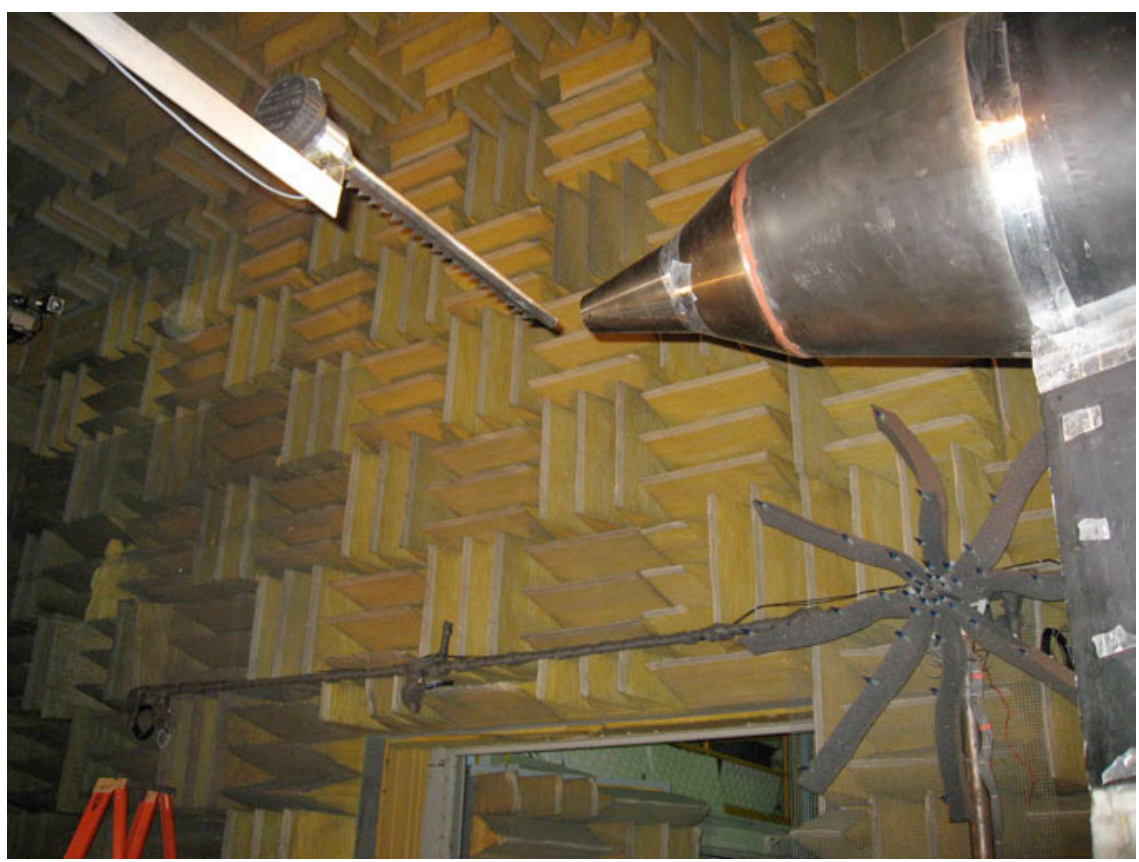

Figure 4. Point noise source mounted 2 inches downstream of the CV Nozzle, Conf. 30000

Phased Microphone Array. Figure 1 shows the JEt noise Directional Array (JEDA) positioned on the rotational arm of the traverse used the study. It is also shown in the background in the photo of Fig. 4, taken during an array "point source" (PS) calibration. The JEDA is comprised of 41 Bruel and Kjaer 4136 (and some equivalent 4939) 1/4-inch free-field microphones mounted in an aluminum frame. The array pattern is designed with a microphone in the center and 5 concentric rings of 8 microphones each. There are azimuthal variations in the microphone positions on each ring, and each ring is double the diameter of the previous ring, working from 
the center outward. The largest ring diameter is 48 ". This array pattern is further discussed in the JEDA Microphone Weighting section. The microphones are held in phenolic sleeves to provide electrical isolation. The entire frame is covered in acoustical foam to reduce installation reflection interference.

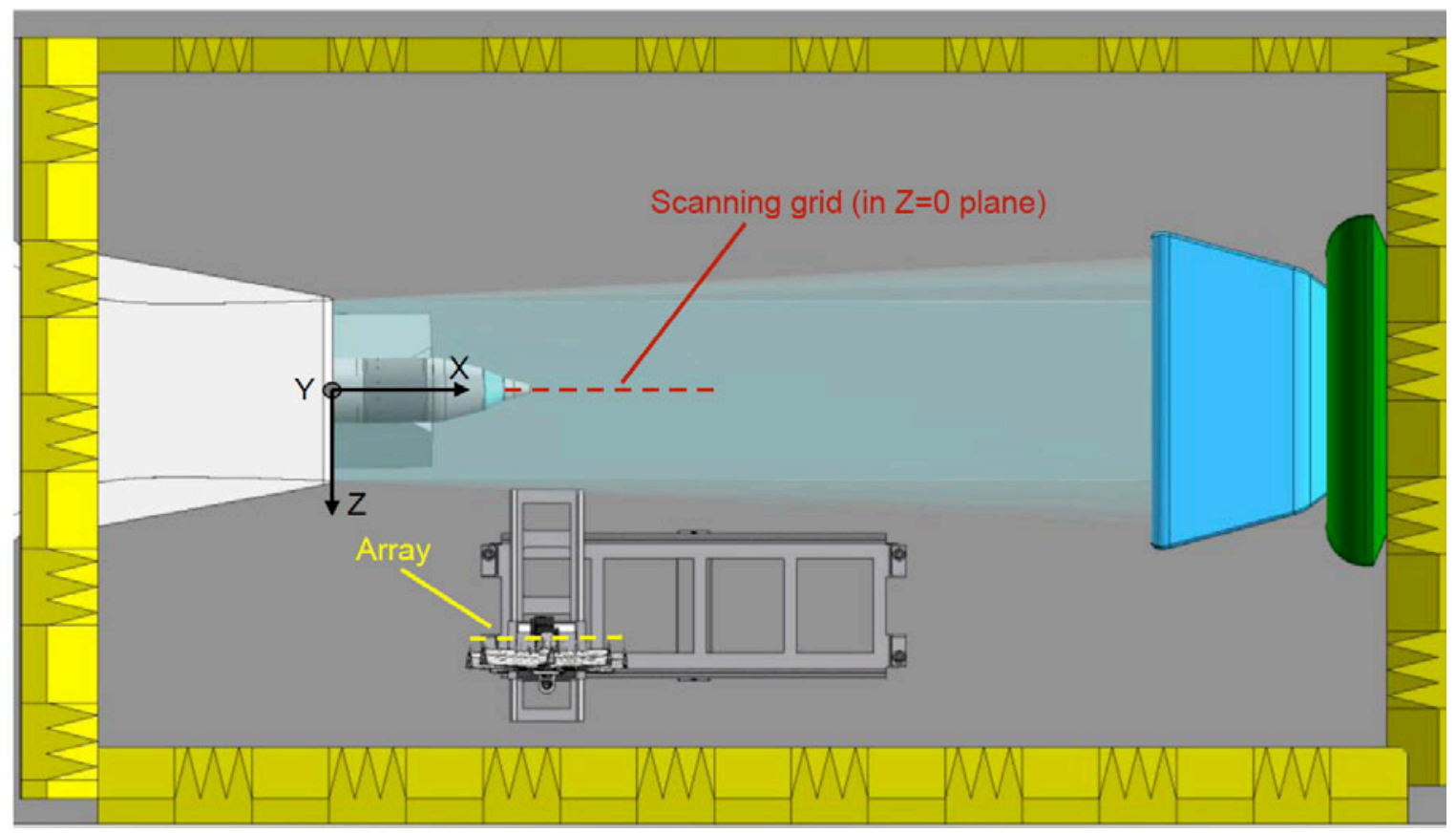

Figure 5. A rotatable JEDA array mounted on X-Z traverse table in the LSAWT. Array is shown positioned at $90^{\circ}$ to jet exit plane. Array microphones and scanning plane locations are indicated.

The JEDA is rotational and is mounted on a $X-Z$ traverse table. The array/jet coordinate system is shown in Fig. 5. The $\mathrm{X}$-axis extends downstream through the nozzle centerline and the positive $\mathrm{Z}$-axis is oriented in the direction of the phased array. The traverse provides a 68 -inch travel distance in the X-direction parallel to the nozzle axis at a constant jet stream sideline distance of $\mathrm{Z}=72$ inches from the nozzle centerline to the center microphone sensing diaphragm. The face of the JEDA can be rotated about the center microphone. The entire traverse table is physically positioned at two different downstream locations in the LSAWT test cell to allow acquisition of phased data at the four stations used in the study. For the present paper, only data from the observation angle of $\theta=90^{\circ}$ are reported, although data were also collected at $\theta=115^{\circ}, 135^{\circ}$, and $150^{\circ}$. The array is set at $X=63^{\prime \prime}$ for all nozzles.

Acoustic Data Acquisition and Initial Processing. Transient data recorders controlled by a workstation were used to acquire the data, simultaneously, from the 41 microphone channels at a sampling rate of $200 \mathrm{kHz}$ recorded in 3 (or 5, depending on test case) separate 15 -second acquisitions. A low pass filter at $80 \mathrm{kHz}$ and a high pass filter at $200 \mathrm{~Hz}$ were used to condition the outputs from each microphone channel. For the present data processing, a crossspectral matrix (CSM) was constructed for each set of data acquired from the JEDA 41 microphone channels. Individual elements of the cross-spectral matrices were computed by partitioning each time signal into 1024 nonoverlapping segments of $2^{13}$ samples. Each time history segment was then Fourier transformed, after using a Hamming window for signal conditioning. The resulting frequency resolution was $24.4 \mathrm{~Hz}$. This cross-spectral representation of the acquired acoustic data is used subsequently in the DAMAS Analysis.

\section{DATA POST-PROCESSING METHOD}

Beamforming and DAMAS. The plan view of the survey (or scan) noise region at and downstream of the jet nozzle is indicated in Fig. 5. The plane of the JEDA array microphone diaphragms is also shown. The test condition is of an open tunnel flow test section with a model jet within the flow and the array on the outside, with a shear layer 
in between. For standard beamforming of the array, the output power spectrum (or response) of the array is obtained from

$$
\mathbf{Y}_{n}=\mathbf{e}_{n}^{T} \mathbf{G e}_{n} / m_{0}^{2}
$$

where matrices are signified by bold letters. This is Eq. (6) from Ref. 3, where here $\mathbf{Y}_{n}=\mathbf{Y}(\mathbf{e})$, when focused at grid point $n$. Here, $\mathbf{G}$ is the Cross-Spectral Matrix (CSM). The total number of microphones in the array is $m_{0}$. The steering "vector" matrix, with respect to a survey grid point at location $n$, is $\mathbf{e}_{n}$ and the component for each microphone $m$ is

$$
e_{m}=a_{m} \frac{r_{m}}{r_{c}} \exp \left(j 2 \pi f \tau_{m}\right)
$$

The steering $e_{m}$ terms account for the amplitude $a_{m}$ and phase changes due to the source distance, flow convection, and refraction through the shear layer to each microphone $m$. The time delay from a grid point to a microphone $m$ is $\tau_{m}$. The calculations employ the use of Snell's law in Amiet's method. 'In Eq. (2), the ratio $r_{m} / r_{c}$ is included to normalize the distance-related amplitude to that of the distance $r_{c}$ from the source location to the array center microphone at $c$. In the method, the emission to observer distance $r_{m}$ is equated to the measured grid-point to observer distance. With this, $r_{m}=\tau_{m} c_{0}$, where $c_{0}$ is the speed of sound of the medium (in the absence of flow).

For the present study, an altered beamform approach is used, with weighting applied to the microphones and the diagonal removed,

$$
\mathbf{Y}_{n}=\frac{\mathbf{e}_{n}^{T} \mathbf{W} \mathbf{G}_{\text {diag }=0} \mathbf{W}^{T} \mathbf{e}_{n}}{\left(\sum_{m=1}^{m_{0}} w_{m}\right)^{2}-\left(\sum_{m=1}^{m_{0}} w_{m}\right)}
$$

where $\mathbf{W}$ is a row matrix containing the terms $w_{m}$. Here $w_{m}$ is a weighting function for the microphones. Also, the CSM matrix $\mathbf{G}_{\text {diag=0 }}$ is formed by zeroing the diagonal terms $G_{m m}$ of $\mathbf{G}$. This reduces the influence of any microphone self noise on the beamform results without negatively impacting subsequent deconvolution operations. ${ }^{3}$

The DAMAS deconvolution algorithm employs beamform results and the steering vectors to obtain apparent source distributions around the noise source region. DAMAS is a method for removing the effects of the array point spread function from the beamformer output, resulting in source presentations which are explicit and straightforward. ${ }^{3}$ After deconvolution, the integrated noise radiated from regions of interest can be determined via a simple summing of mean-squared pressures over each chosen region.

JEDA Microphone Weighting. Previous DAMAS application studies ${ }^{12,13,14}$ for airframe noise in Langley's Quiet Flow Facility (QFF) have employed microphone weighting (or shading) such that Eq. (3) produced constant beamforming resolution over set ranges of frequencies. This was done by shading out $\left(w_{m}=0\right)$ inner microphone groups at low frequencies and by shading out outer groups at high frequencies. (This is in contrast with setting all $w_{m}=1$ and making all microphones fully active at all frequencies.) For the QFF tests, this weighting was found useful for the model size and the test setups tested. The JEDA was designed with this approach in mind.

However, for the present jet noise data, it was found that an alternate weighting is preferred - one that more effectively reduces undesirable beamform side lobes across intense jet noise regions, therefore producing cleaner DAMAS results. Although DAMAS accounts for side lobes, reducing side lobe intensity gives a signal-to-noise advantage. The weighting chosen is linear with the center microphone (Mic 1) at $w_{1}=0.1$ and all microphones on the outer ring at $w_{m_{0}}=1$. In general,

$$
w_{m}=w_{1}+\left(1-w_{1}\right)\left(R_{m} / R_{m_{0}}\right)
$$


where $R_{m}$ is the array radial distance from the center Mic 1 to the Mic $m$. All microphones on the outer ring are at $R_{m_{0}}=24$ ". Lastly, note that $w_{m}$ is not a function of frequency.

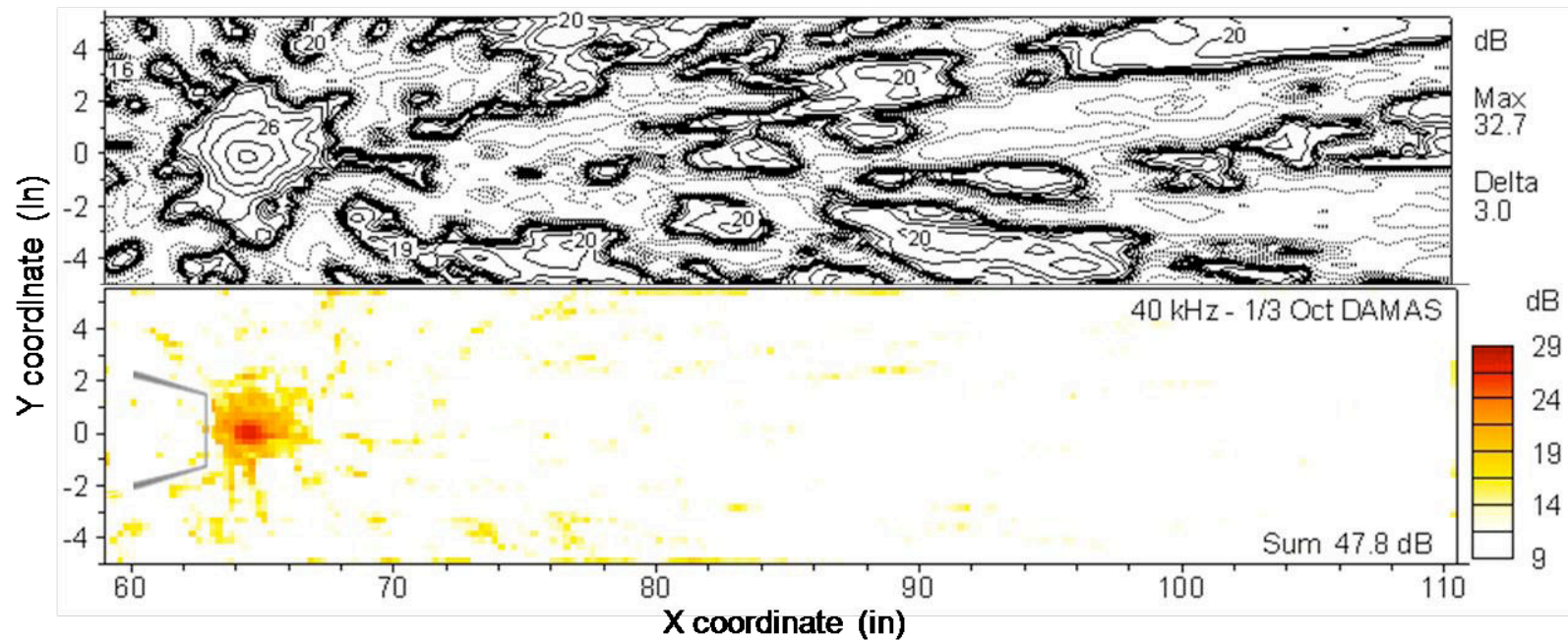

Figure 6. Results for a calibration PS at $(X, Y)=(65 ", 0 ")$. Wind tunnel flow at $M_{W T}=0.1$. Jet flow is at zero velocity. $40 \mathrm{kHz} \mathrm{1/3-octave} \mathrm{band} \mathrm{is} \mathrm{shown} \mathrm{for} \mathrm{250-iteration} \mathrm{DAMAS} \mathrm{with} \mathrm{no} \mathrm{CSM} \mathrm{Conditioning.} \mathrm{Beamform} \mathrm{(top)} \mathrm{and} \mathrm{DAMAS}$ (bottom) is presented.

Steering Vector $\mathbf{e}_{n}$ Calibration. The success of DAMAS to define a proper presentation of a noise source distribution depends on the accuracy of the steering vector $\mathbf{e}_{n}$, as well as the correctness of the assumption of incoherence between noise sources over the region. For the determination of $a_{m}$ and $\tau_{m}$ in the steering vector terms $e_{m}$ of Eq. (2), the Amiet ${ }^{11}$ method assumes that acoustic rays from each source are convected downstream in a uniform velocity flow field, refracted at an infinitely thin shear layer, and then propagated in a quiescent medium to the observer (microphone). For beamforming and DAMAS applications, the added effect of curved wind tunnel flow has been incorporated into the steering vector terms ${ }^{13,15}$. However, there has been no accounting of the actual thick shear layers that have varying velocity gradients. (Amiet's thick shear layer relation affects the amplitude $a_{m}$, but does not address any time transmission. Thus, phase differences that would occur compared to a thin shear layer model is not addressed.)

For the point source (PS) calibration configuration of Fig. 4, a beamform dB level contour presentation is given in Fig. 6 for the $40 \mathrm{kHz} 1 / 3$-octave band. The dB levels are $10 \log \left(Y_{n}\right)$ over the $n$ grid points. The "negative" dB levels, shown as dashed curves, are $-10 \log \left|Y_{n}\right|$, when $Y_{n}$ is negative due to the diagonal removal operation of Eq. (3). The "PS" is the open end of a 0.75 " ID tube that is connected to a speaker-driver. The array is 72 " away. The grid window size is 52" by 10", with grid point separation of $\Delta x=0.25$ ". The test case is for the jet turned off, and the PS is emitting broadband noise in the presence of wind tunnel flow of $M_{W T}=0.1$. The PS center is positioned 2" downstream of the nozzle exit. (The irregular beamform pattern is due to the irregular microphone pattern of the array.) The corresponding DAMAS processed result is shown in Fig. 6. The dynamic range used is $20 \mathrm{~dB}$. It is seen that the source location is shifted in the upstream direction by about 0.5 ". The DAMAS image appears somewhat blurred, but keeps the source center well defined within the PS tube opening. The source location shift is primarily due to array misalignment rather than the tunnel flow because the PS location, for the $M_{W T}=0$ case, was found to be shifted in the same way, but the image burring was much less for the $M_{W T}=0$ case. (This is shown subsequently in Figs. 9-12.)

The calibration test cases, such as that shown in Fig. 6, are used in the present paper as a basis for improving jet noise imaging. In the present study, a method is developed to correct phase effects, whether due to shear layer effects or by traverse/array positioning errors. Amiet's amplitude corrections are presumed sufficiently accurate for present purposes. (It could be argued that to be complete, one must account for amplitude error. While this is acknowledged, such errors are taken as having a smaller effect compared to that of the phase effects. Amplitude corrections to Amiet's method are thus beyond the scope of this paper.) 
$\mathbf{e}_{n}$ Calibration: CSM Phase Conditioning. For the present study, rather than correct $\mathbf{e}_{n}$ per se, we "condition" the CSM cross-spectrum $\mathbf{G}$, as it is more directly accomplished with point source (PS) calibration data. A calibration PS is placed at location $n$ in the region where such calibrations would be applied. The array is in a fixed location, and the jet source is stopped. If the measured individual terms of $\mathbf{G}$ for the point source location are $G_{i j}=\left|G_{i j}\right|_{p s} \exp \left(j\left(\varphi_{i j}\right)_{p s}\right)$ and the predicted phase for the point source location $n$ is $\left(\varphi_{i j}^{\prime}\right)_{p s}$, Then $\left(\varphi_{i j}^{\prime}-\varphi_{i j}\right)_{p s}$ represents an error-offset in phase for the array microphone pair, $i j$. For example, plot showing measured crossspectral phase term $\varphi_{1,29}$ from $\mathbf{G}$ is shown in Fig. 7 (a). Microphone 1 is at the array center and Microphone 29 is on the fourth ring. The predicted $\left(\varphi_{1,29}^{\prime}\right)_{p s}$ is shown offset from $\left(\varphi_{1,29}\right)_{p s}$. Then for a model test case, a conditioned $\mathbf{G}$ would have the terms

$$
\left(G_{i j}\right)_{c o n d}=\left|G_{i j}\right| \exp j\left(\varphi_{i j}+\left(\varphi_{i j}^{\prime}-\varphi_{i j}\right)_{p s}\right)
$$

where $\varphi_{i j}$ is the test case phase. (Note that microphone 29 was chosen because the experimental phase with microphone 1 was most offset from the theory. This extreme may best illustrate the method. Microphone 29 could have been misaligned during set up.) An assumption of using Eq. (5) in beamforming is that the particular microphone pair error offsets are the same for all $n$ grid points in the survey region. This should be approximately valid whenever the array and the source region to be surveyed are small compared to the distance between them. Over such regions, the ray path orientations to the shear layer would be, to the first order, equal, and thus give generally the same phase errors relative to each microphone pair. In beamforming, steering vectors would be unchanged from what would be otherwise used.

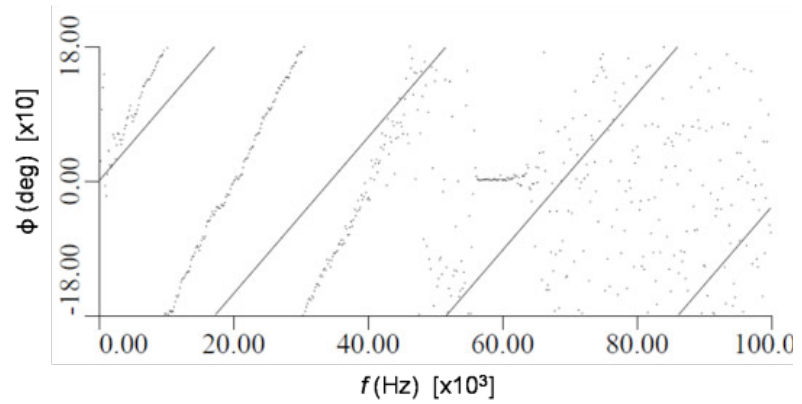

(a) Cross-spectral phase data with $\varphi_{1,29}$ prediction (straight line segments)

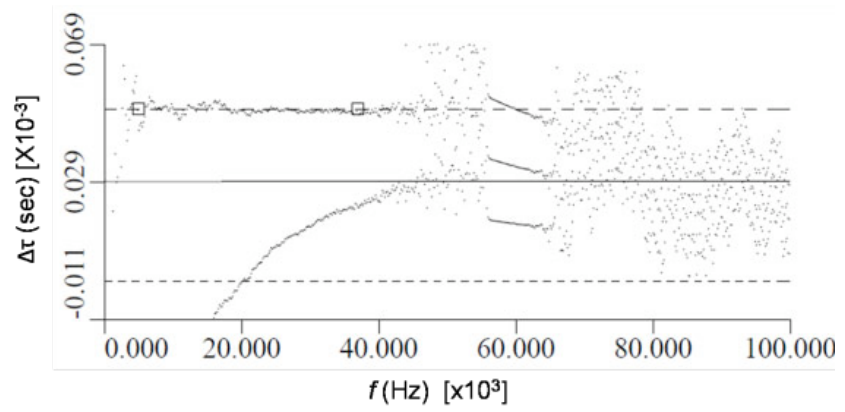

(c) Signal time delay from cross-spectral phase, $\Delta \tau_{1,29}$ (dashed line) prediction (straight solid line), delay off-set found from high coherence range

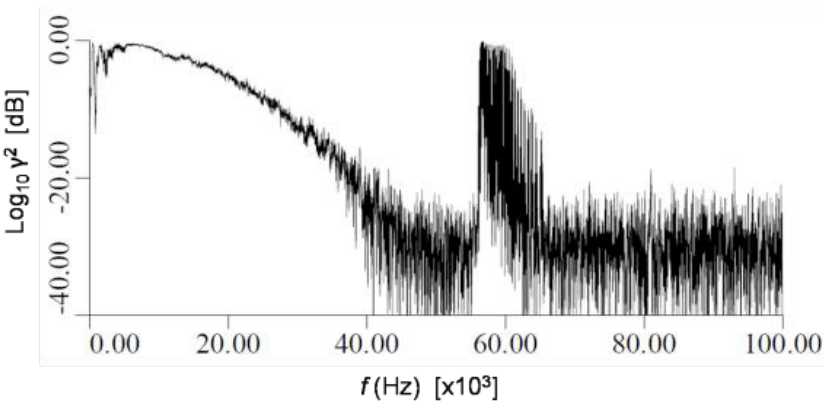

(b) Coherence $\gamma_{1,29}^{2}$ in $\mathbf{d B}$

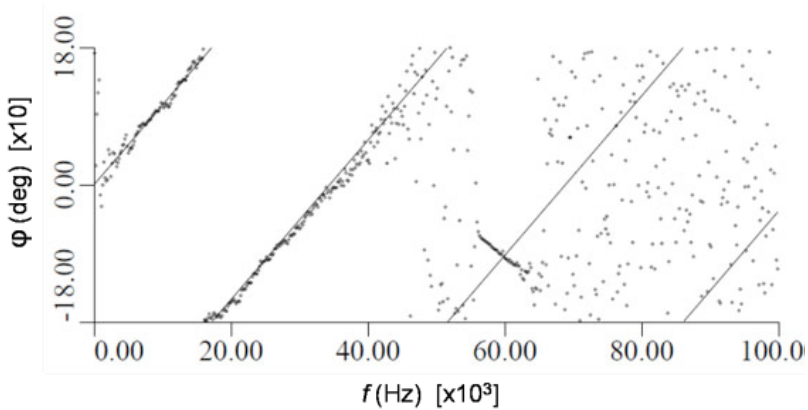

(d) Conditioned phase data with prediction

Figure 7. Cross-Spectral phase and coherence for term $G_{1,29}$, prediction, and conditioned phase offsets 


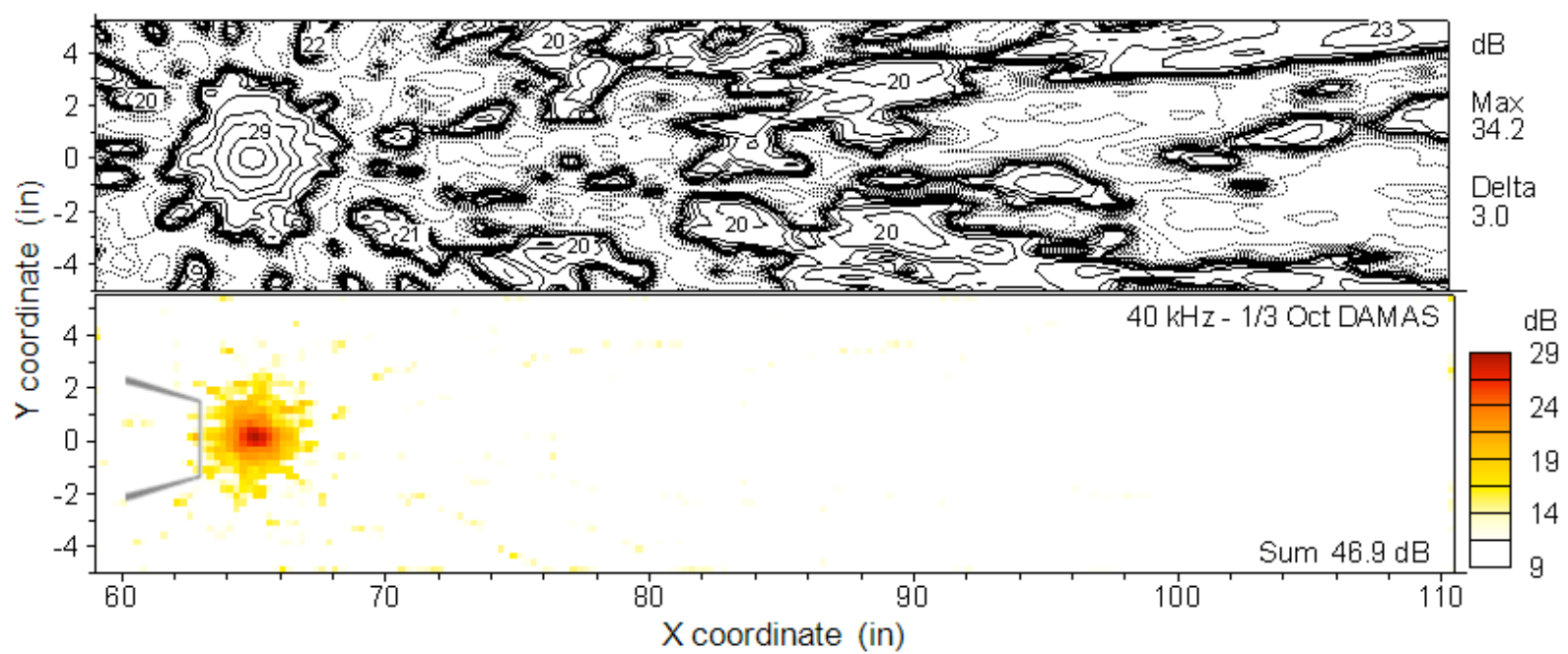

Figure 8. Results for the PS calibration case of Fig. 6, but for CSM Conditioning

Equation (5) indicates that if the experimental test case $\mathbf{G}_{\text {meas }}$, being processed, is the calibrating PS case itself, then the $\mathbf{G}_{\text {cond }}$ phases become simply $\left(\varphi_{i j}^{\prime}\right)_{p s}$. Since these phases match the specified point source location at a grid point on the scanning plane, any beamforming using these steering vector phases would result in a near perfect point spread function, centered about the specified source location. Note that even if the source-location grid point were not at the actual source location, the specification of location itself in calculating $\left(\varphi_{i j}^{\prime}\right)_{p s}$ would make the image shift in beamforming and DAMAS to match the specified location. The corresponding DAMAS would give a nearly perfect source location result. This would not be exact because the cross-spectral amplitude $\left|G_{i j}\right|$ would not equal that of an exact case.

$\mathbf{e}_{n}$ Calibration: CSM $\tau$ Phase Conditioning. The above conditioning method is not effective outside the range of strong PS output, where the PS dominates extraneous sources. The coherence for the microphones is given in Fig. 7 (b). It is seen that the PS dominates from about $5 \mathrm{kHz}$ through $40 \mathrm{kHz}$. A modified method is defined here that first determines the mean values of PS time delay between the array microphone pairs. Then, this is used to condition the full frequency range of the experimental case CSMs.

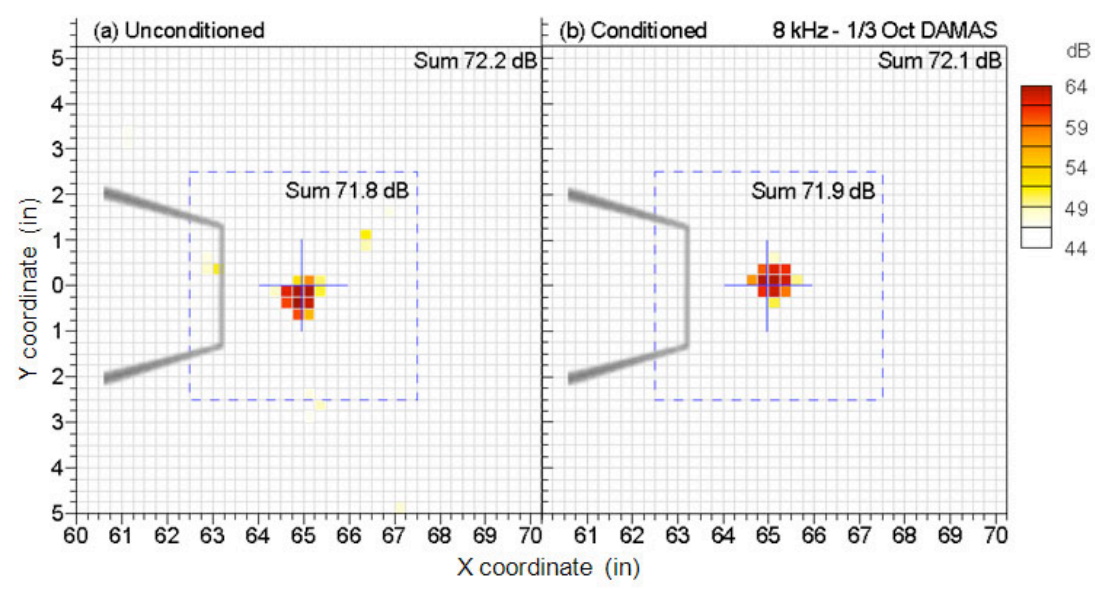

Figure 9. Processed results for the no-windtunnel-flow PS calibration case for the $8 \mathrm{kHz}$ 1/3-octave band. (a) no CSM Conditioning and (b) with CSM Conditioning 


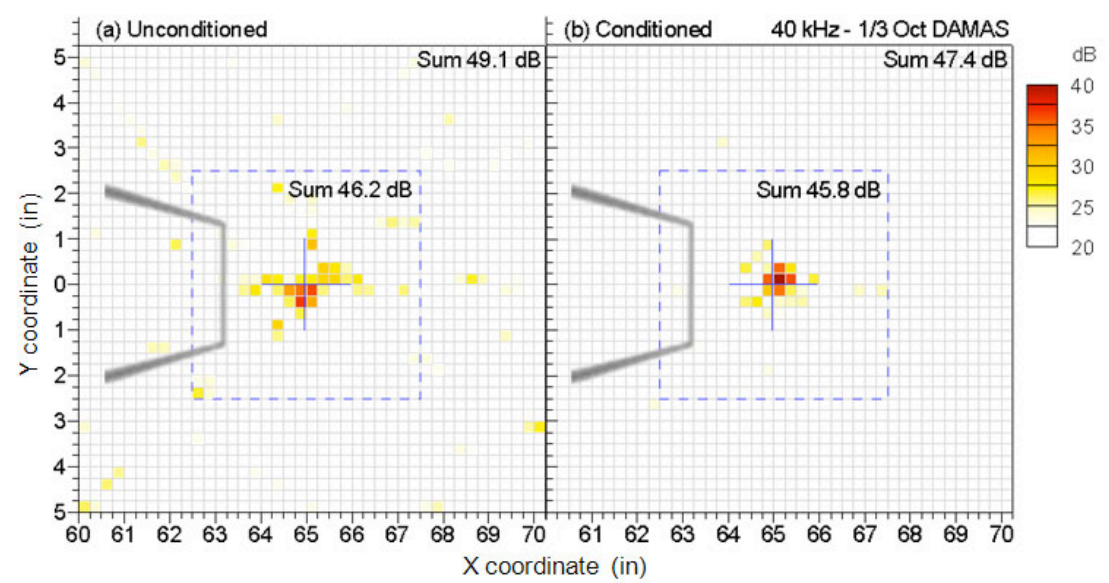

Figure 10. Processed results for the no-windtunnel-flow PS calibration case for the $40 \mathrm{kHz} 1 / 3$-octave band. (a) no CSM Conditioning and (b) with CSM Conditioning

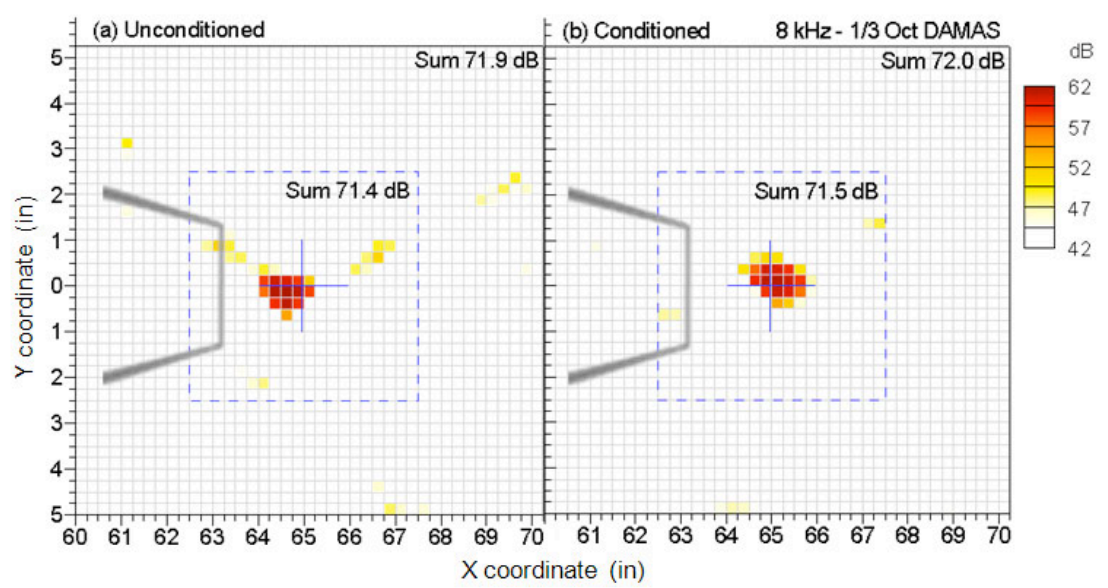

Figure 11. Processed results for the $M_{W T}=0.1$ PS calibration case for the $8 \mathrm{kHz} 1 / 3-$-octave band. (a) no CSM Conditioning and (b) with CSM Conditioning.

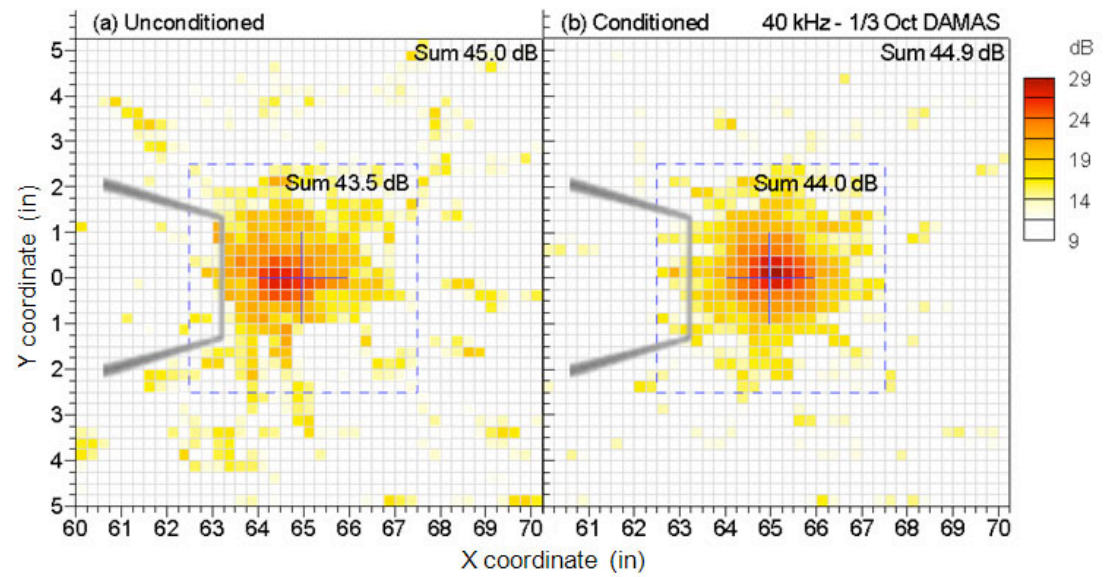

Figure 12. Processed results for the $M_{W T}=0.1$ PS calibration case, for the $40 \mathrm{kHz} 1 / 3$-octave band. (a) no CSM Conditioning and (b) with CSM Conditioning 
This method determines a $\mathbf{G}_{\text {cond }}$ where the terms

$$
\left(G_{i j}\right)_{c o n d}=\left|G_{i j}\right| \exp j\left(\varphi_{i j}+\left(\varphi_{i j}^{\prime}-\bar{\varphi}_{i j}\right)_{p s}\right)
$$

The phase angle $\left(\bar{\varphi}_{i j}\right)_{p s}$ is based on a mean value of time difference it takes to propagate from the point source grid location to microphone $i$, compared to microphone $j$. The microphone phases are taken as

$$
\varphi_{i}=2 \pi f \tau_{i} \text { and } \varphi_{j}=2 \pi f \tau_{j}
$$

The time difference is

$$
\Delta \tau_{i j}=\tau_{j}-\tau_{i}=\left(\varphi_{i j}+k 2 \pi\right) / 2 \pi f
$$

where $k$ takes on values $0,1,2, \ldots$ There is normally an obvious value for $k$ based on $\left(\varphi_{i j}^{\prime}\right)_{p s}$ and frequency ranges of strong PS coherence. An average value of $\Delta \tau_{i j}$ is found by taking into account the coherence between the two microphone channels

$$
\Delta \overline{\boldsymbol{\tau}}_{i j}=\sum_{n=n_{\ell}}^{n=n_{u}} \Delta \tau_{i j}(f) \gamma_{i j}^{2}(f) \Delta f / \sum_{n=n_{\ell}}^{n=n_{u}} \gamma_{i j}^{2}(f) \Delta f
$$

where $f=n \Delta f, n=0,1,2, \ldots$, and

$$
\gamma_{i j}^{2}(f)=\left|G_{i j}^{*} G_{i j}\right|^{2} / G_{i i} G_{j j}
$$

is the coherence, which for microphone 1 and 29 is shown in Figure 7 (b). Then, the phase angle

$$
\left(\bar{\varphi}_{i j}\right)_{p s}=2 \pi f \Delta \bar{\tau}_{i j}
$$

Criteria are needed to establish ranges of upper and lower frequencies where averaging is done. For the present data, we chose $n_{\ell}$ and $n_{u}$ to be the lower and upper bin numbers that would give $f_{\ell} \geq 5 \mathrm{kHz}$ and $f_{u}$ to be where mean values of $10 \log \gamma_{i j}^{2}(f)$ over a sliding frequency range of $500 \mathrm{~Hz}$ drop below $-20 \mathrm{~dB}$. An added criterion for $f_{u}$ is that the standard deviation of $10 \log \gamma_{i j}^{2}(f)$ is not more than $4 \mathrm{~dB}$ over that band. When these criteria are exceeded, averaging is stopped. These criteria and Eq. (8) lead to Fig. 7(c), where it shows $\Delta \tau_{1,29}$, calculated from $\varphi_{1,29}$, the predicted $\Delta \tau_{1,29}^{\prime}$, and the $\Delta \overline{\boldsymbol{\tau}}_{1,29} . \Delta \bar{\tau}_{1,29}$ is shown to be constant over the frequency range (5 $\mathrm{kHz}$ to almost $40 \mathrm{kHz}$ ) indicated. Figure $7(\mathrm{~d})$ shows $\varphi_{i j}^{\prime}$ compared to the conditioned $G_{1,29}$ phase angle $\left(\varphi_{1,29}+\left(\varphi_{1,29}^{\prime}-\bar{\varphi}_{1,29}\right)_{p s}\right)$. Such $\Delta \overline{\boldsymbol{\tau}}_{i j}$ values are used in Eq. (11) to get $\left(\varphi_{i j}^{\prime}-\bar{\varphi}_{i j}\right)_{p s}$ for Eq. (6) beamforming.

The corresponding beamform and the DAMAS solutions, using $\mathbf{G}_{c o n d}$, are shown in Fig. 8. The angles $\left(\bar{\varphi}_{i j}\right)_{p s}$ are used for all $n$ in the scanning plane for jet noise cases with matching experimental setups. (At times, PS data were not obtained after the traverse was moved. In such cases, a non-matched case is used and the image positions can be offset incorrectly.) Figure 8 shows almost the same scatter as Fig. 6 for the unconditioned beamform and DAMAS, but the pattern is properly positioned (at $X=65$ ”), more symmetrical, and the strength at the center is higher. The scatter is due to the sound passing through turbulence on the way to the array microphones. A corresponding drop in coherence (signal-to-noise) is seen in the $40 \mathrm{kHz}$ band. This scatter was dealt with in Refs. 15 and 3. One would anticipate that such a degree of scatter would result for the jet noise cases also.

For the $1 / 3$-octave frequency band of $8 \mathrm{kHz}$, Fig. 9 shows no-CSM-conditioned and CSM-conditioned results for the no wind tunnel flow, $M_{W T}=0$, PS calibration case. The presentation window size is reduced, but DAMAS 
processing was done for the same window size as that of Figs. 6 and 8. Figure 10 has the $40 \mathrm{kHz}$ band plots for this no-flow case. The scatter is seen to be small and images are generally confined to the PS tube's inside diameter of 0.75 ". The $M_{W T}=0.1$ cases for the same two frequencies are shown in Figs. 11 and 12 . The $40 \mathrm{kHz}$ band results, of course, correspond to Figs. 6 and 8. The scatter for the lower frequency band is seen to be much less than that of the higher band. In the figures, a cross is placed at the center of the PS position. The conditioning brings the image back to the PS center. (Note, the color in each block corresponds to the level at the grid point at the lower left grid line intersection.) The summed $\mathrm{dB}$ levels in each window are seen to be approximately the same between the unconditioned and the conditioned CSM when the PS region is isolated. This illustrates that the sum about a source region is correctly determined even if the array "focus" is not perfect.

$\mathbf{e}_{n}$ Calibration: Ambient Temperature Conditioning. Equation (5) is taken as valid when the ambient temperatures of the test point and PS test are the same. If the temperatures of the PS and the test are different, say absolute $T_{\text {test }}$ instead of $T_{p s}$, then one needs to correct for the likely difference in the phase. Since the phase prediction is $\varphi_{i j}^{\prime}=2 \pi f \Delta r_{i j} / c$, where $\Delta r_{i j}=r_{i}-r_{j}$, and $c$ is the speed of sound $\approx \sqrt{T}$, the temperature-corrected $\mathbf{G}_{\text {cond }}$ terms are, for $T_{\text {test }}$,

$$
\left(G_{i j}\right)=\left|G_{i j}\right| \exp j\left(\varphi_{i j}+\left(\varphi_{i j}^{\prime}-\bar{\varphi}_{i j}\right)_{p s, T p s} \sqrt{T_{p s} / T_{t e s t}}\right)
$$

\section{RESULTS}

This section presents example jet noise data that was processed employing the methodology of the last section. The CSM for each calibration and jet noise test case had a frequency resolution of $24.4 \mathrm{~Hz}$. The beamforming, DAMAS, and conditioning of each is accomplished for the CSM at a $171 \mathrm{~Hz}$ bandwidth, obtained by summing vectorially seven $24.4 \mathrm{~Hz}$ bands. Conditioning is performed using Eq. (12). The 1/3-octave band and full-octave band maps (spatial plots) of noise source grid window levels are obtained by summing, on a pressure-squared basis, $171 \mathrm{~Hz}$ band maps.

DAMAS Noise Distribution and Spectra. Figure 13 shows the 1/3-octave DAMAS results for the cold-jet single convergent (CV) nozzle of Fig. 2 (a). The core Nozzle Pressure Ration (NPR) was 1.52, jet Mach number $M_{J}=0.8$, jet stagnation temperature $T_{J}=100^{\circ} \mathrm{F}$, no wind tunnel flow $M_{W T}=0$, with the temperature of the test section at $T_{\text {test }}=88.2^{\circ} \mathrm{F}$. This condition was used to examine cold subsonic mixing noise distributions. In Fig. 13 , the $f_{1 / 3}=8 \mathrm{kHz}$ band shows that noise emission is strongest from one to three nozzle diameters (D) downstream from the nozzle. There seems to be a concentration appearance of noise along the nozzle lip near the nozzle exit, as viewed from the side. This characteristic is also seen for $f_{1 / 3}=40 \mathrm{kHz}$. Also, at this higher frequency, the noise concentrates more strongly closer to the nozzle.

We know the jet should be symmetrical, but there appear to be some non-symmetrical source distributions for 8 $\mathrm{kHz}$. This should be traceable to DAMAS generation subtleties. One could suggest that, at this frequency, it is an inaccurate assumption that the sources can be cast purely in terms of an incoherent distribution of sources. For strong coherence, one would expect the image to have a fractured appearance and a stacking of "sources" around the edges of the scanning plane. There is only a little evidence of that for $8 \mathrm{KHz}$ in Fig. 13. Part of the non-symmetric appearance should be due to a non-symmetrical beamforming sidelobe pattern (not shown), which DAMAS employs, but is cut off at the top of the scanning plane. To examine this, a DAMAS run was made after redefining the grid pattern to capture this. Some improvement in image balance was found. (These possible reasons for nonsymmetry apply to all images that follow in this paper.) A larger degree of symmetry is found in Fig. 13 for the $f_{1 / 3}=40 \mathrm{kHz}$ DAMAS plot. At this higher frequency, it appears that all criteria for DAMAS applicability are met for the jet. Generally at $8 \mathrm{kHz}$, the criteria appear met. 


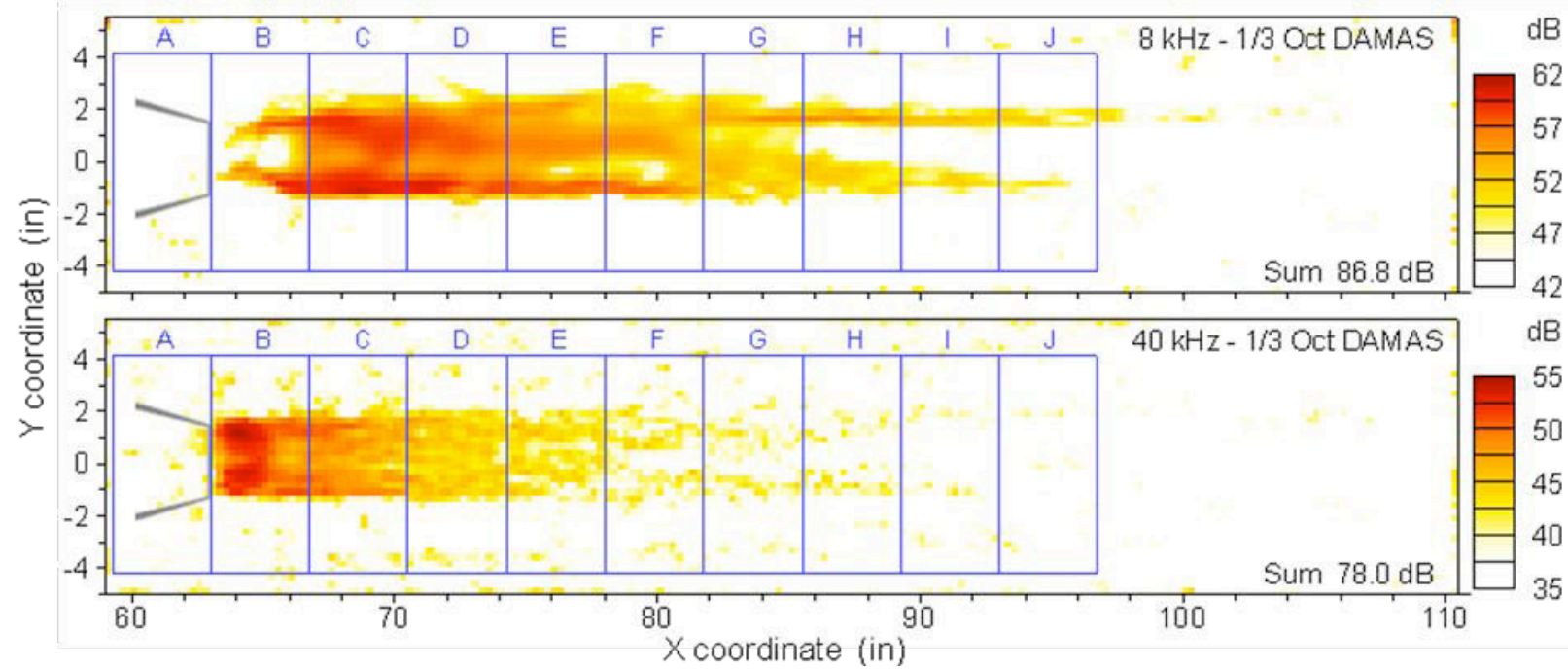

Figure 13. DAMAS results for single CV nozzle for subsonic cold jet at $M_{J}=0.8$ and no wind tunnel co-flow, $M_{W T}=0$. Both 8 and $40 \mathrm{kHz}$ 1/3-octave are presented.

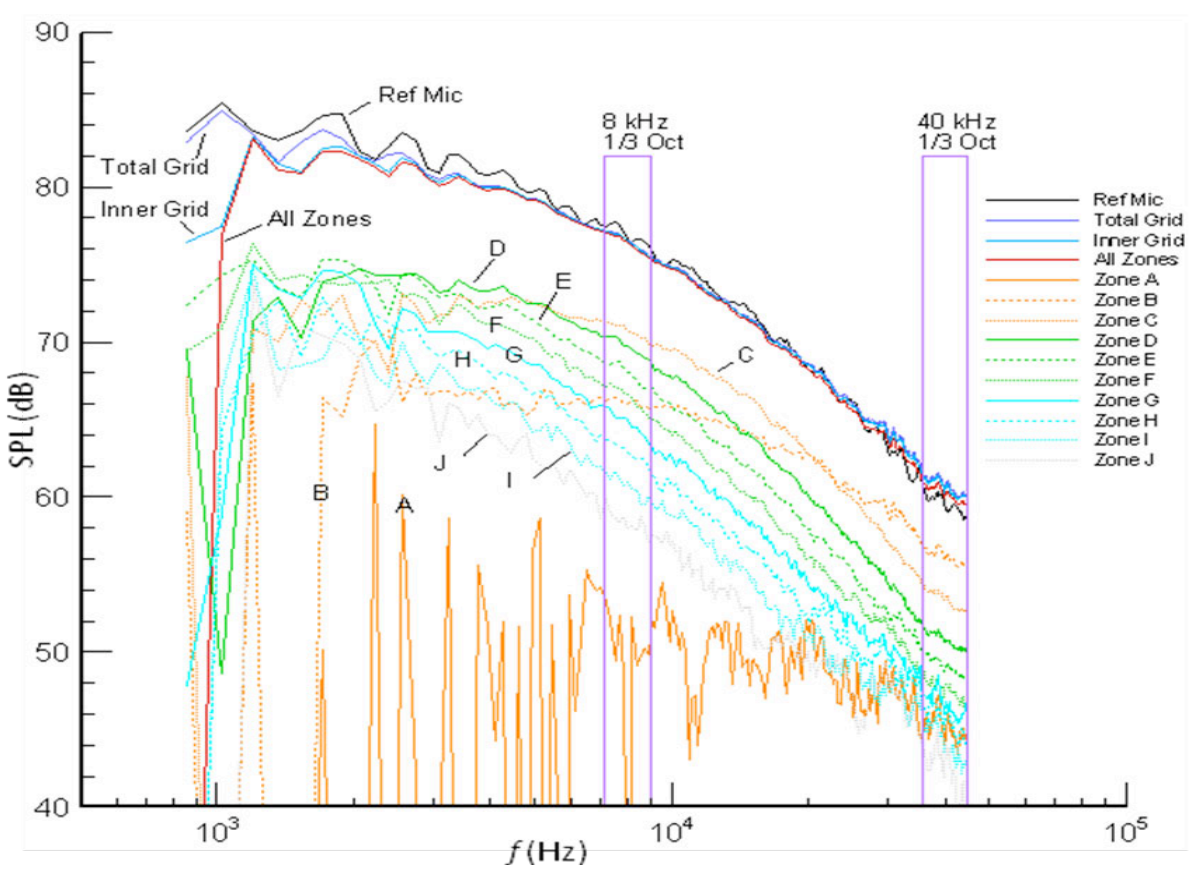

Figure 14. Spectra over spatial regions for the CV-nozzle jet noise defined in Fig. 13. The bandwidth of the data is $141 \mathrm{~Hz}$. The 1/3-octave regions of Fig. 13 are shown located.
In Fig. 13 for both frequencies, the DAMAS maps over the noise regions are separated into zones A through J. These zones are used to define where noise source distributions are integrated to determine contributions to the total noise spectrum at the array observer position. For DAMAS, integration is accomplished by simple pressure-squared summing over grid points in the zones. Figure 14 shows, for both frequencies, spectra attributable to each zone. The spectra have $171 \mathrm{~Hz}$ bandwidth levels plotted on a $\log$ scale. The individual zone spectra are added to produce a total from all zones as indicated. This zone total is seen to be very close to the total from all grid points, except for the border and is close to the reference microphone spectrum from an individual microphone of the array (a microphone in the third ring is used). It suggests, when the sum matches the auto-spectrum of the single microphone, that most of the noise energy distribution can be modeled as incoherent.

Figure 15 shows, for the same CV-nozzle, DAMAS results for a supersonic condition at $M_{J}=1.48$. The core NPR $=3.57$, the $T_{J}=100^{\circ} \mathrm{F}$, no wind tunnel flow $M_{W T}=0$, and $T_{\text {test }}=88.3^{\circ} \mathrm{F}$. This condition was used to examine cold under-expanded broadband shock noise distributions. In Fig. 15 , the $f_{1 / 3}=8 \mathrm{kHz}$ band shows that noise 


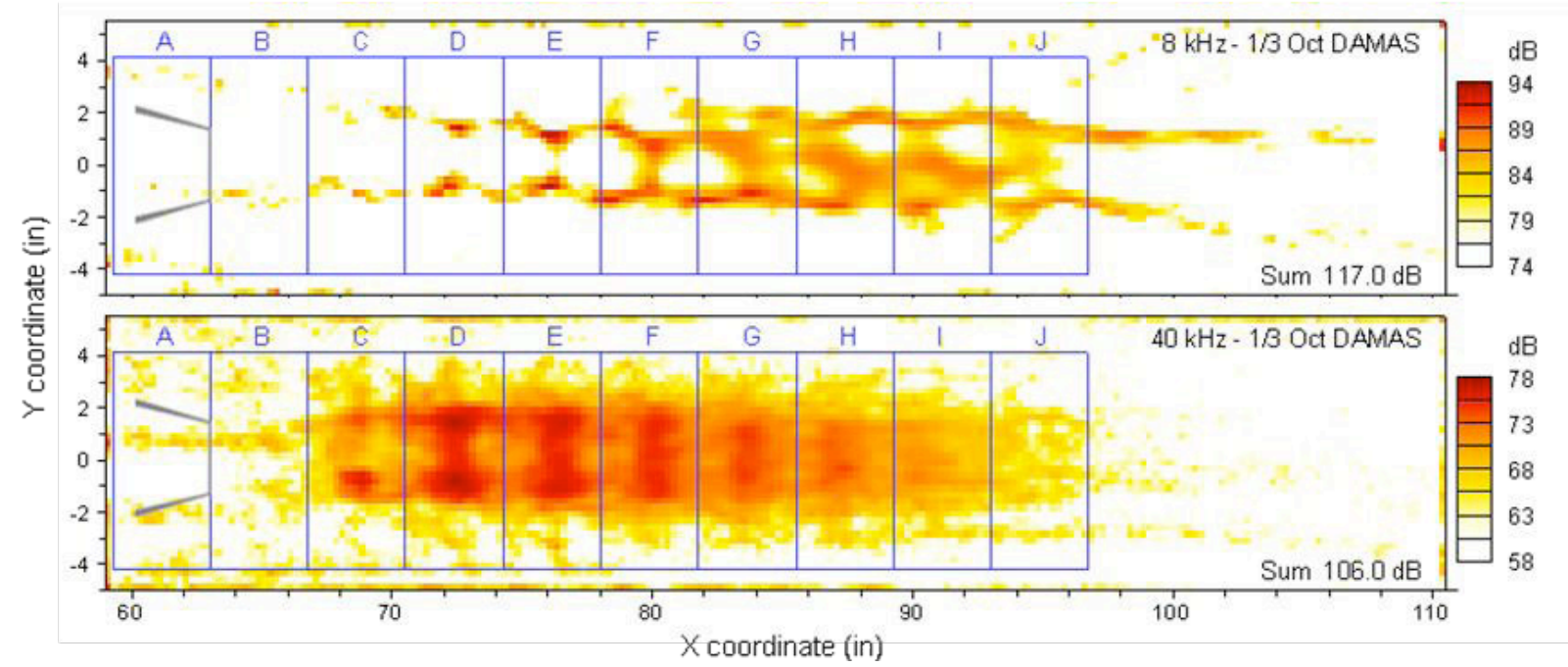

Figure 15. DAMAS results for single $C V$ nozzle for supersonic cold jet at $M_{J}=1.48$ and no wind tunnel co-flow, $M_{W T}=0$. Both 8 and $40 \mathrm{kHz}$ 1/3-octaves are presented.

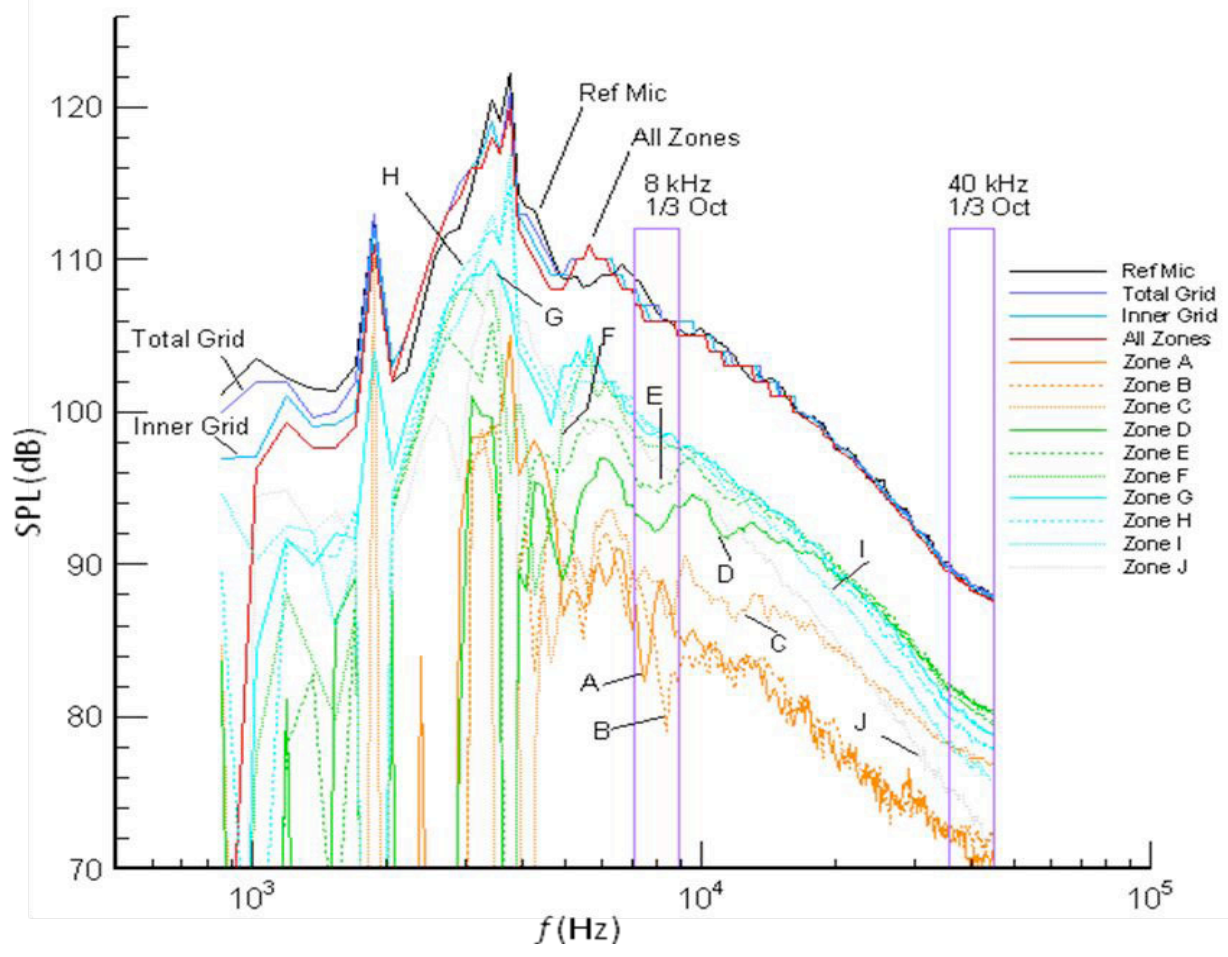

Figure 16. Spectra over spatial zones (or regions) for the $\mathrm{CV}$-nozzle jet noise defined in Fig. 15. The bandwidth of the data is $141 \mathrm{~Hz}$. The 1/3-octave regions of Fig. 15 are shown located.

J of Fig. 15. Strong screech tones are seen at 1.8 and $3.6 \mathrm{kHz}$. The low frequency end of the $8 \mathrm{kHz} 1 / 3-\mathrm{octave}$ band is near the side of a broadband peak centered about three times $1.8 \mathrm{kHz}$. It is likely that a screech tone contribution affects the $8 \mathrm{kHz}$ 1/3-octave band image in Fig. 15. The $40 \mathrm{kHz}$ band shows very good agreement between the relative levels. diameters downstream from the nozzle. Little noise from the nozzle lip region is apparent. Although this band is beyond the obvious screech frequencies, there may be coherent noise contributions into the frequency band. These may cause some of the split shock-cell structure appearance. Some high levels around the grid window border are also noted. Such features are not seen in the $f_{1 / 3}=40$ $\mathrm{kHz}$ case in Fig. 15. This figure also shows maximum contributions are several diameters forward of the lower frequency case distribution.

Figure 16 shows the spectra associated with the

emission appears strongest from five for so nozzle

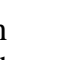

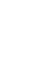

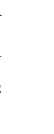

.


Single-Jet Nozzle Configurations. In this section, DAMAS results are given in full-octave presentations in order to represent more of the total energy in the spectra than did the $1 / 3$-octave maps. The $31.5 \mathrm{kHz}$ full-octave band includes the 1/3-octave $40 \mathrm{kHz}$ band. Figures 17 and 18 show octave results for the same jet cases of Figs. 13 and 15. Except for increased levels over the jet regions, there are little basic differences in the presentations or interpretations.

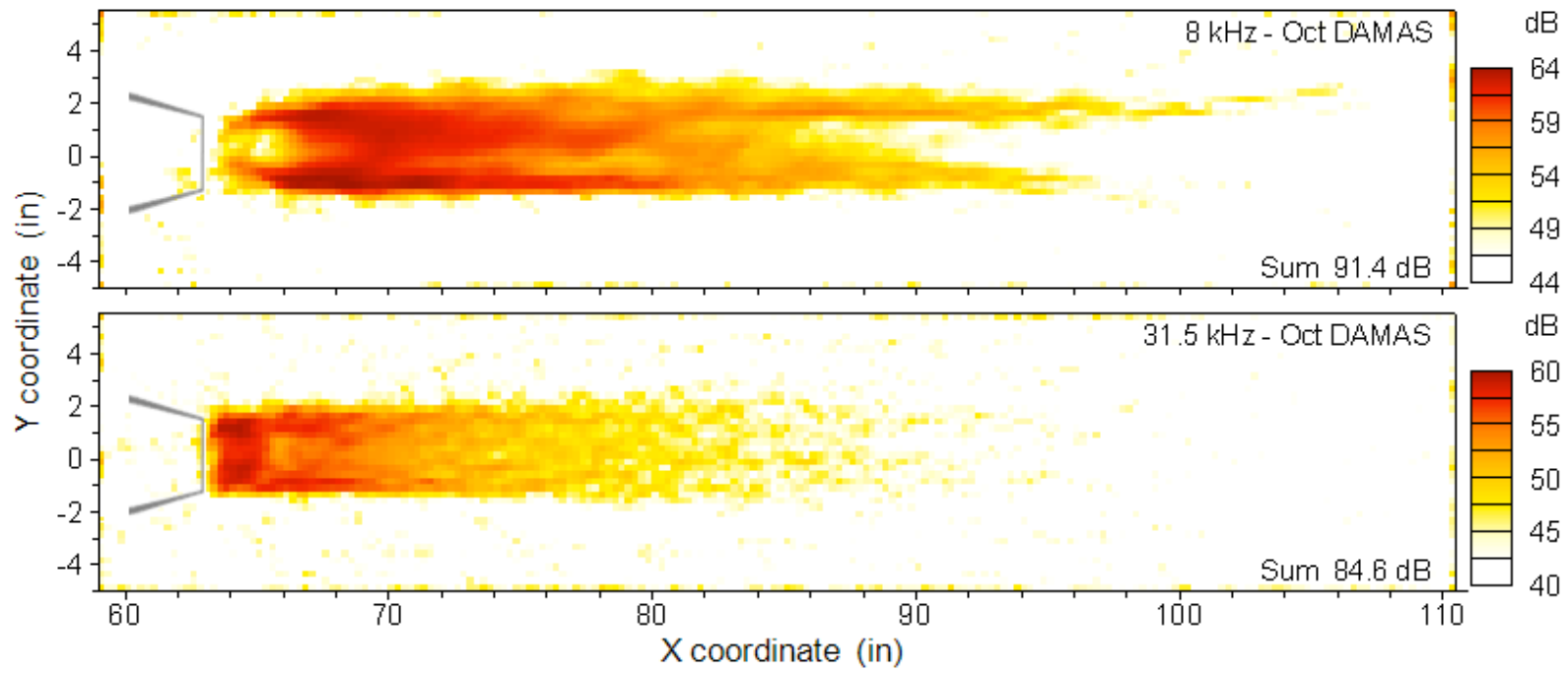

Figure 17. Single CV nozzle for subsonic cold jet at $M_{J}=0.8$ and no-wind tunnel co-flow, $M_{W T}=0$. Both 8 and 31.5 kHz octave presented.

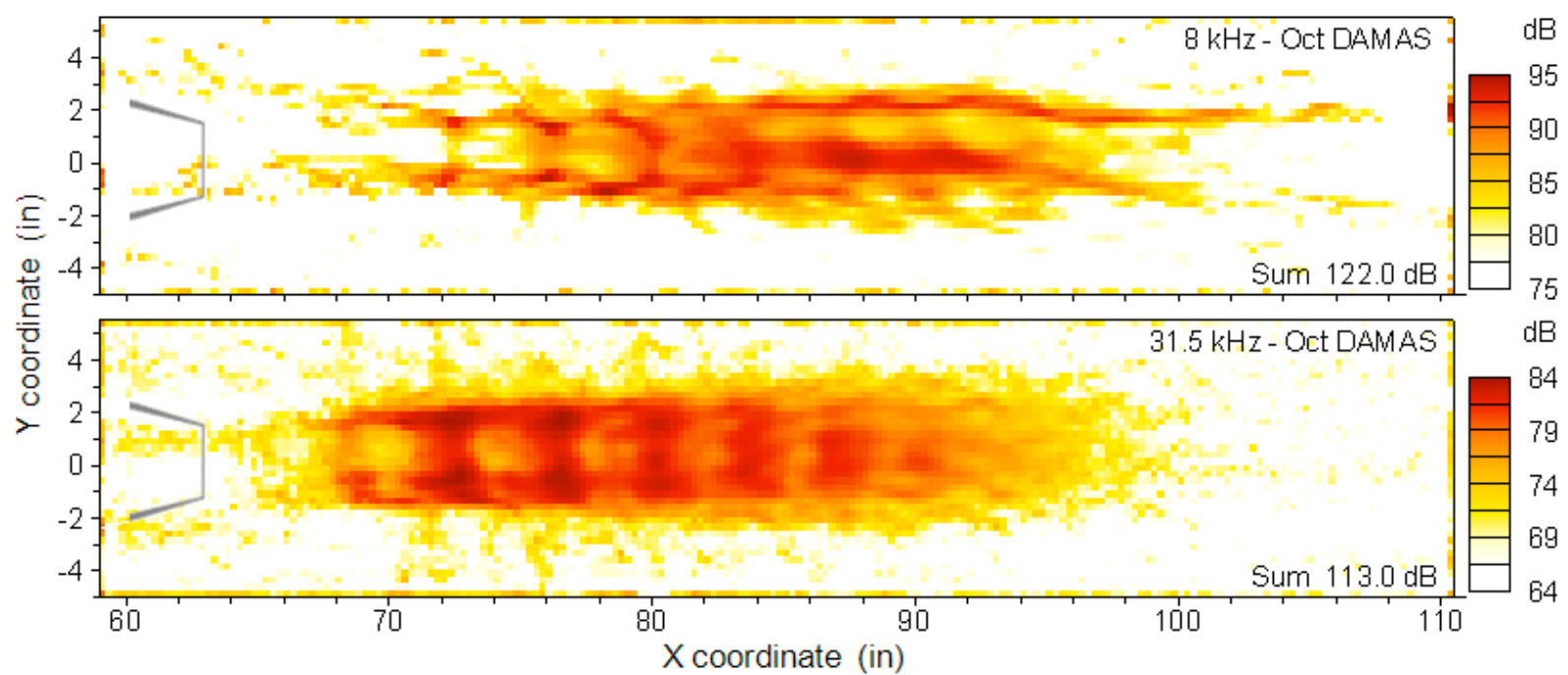

Figure 18. Single CV nozzle for supersonic cold jet at $M_{J}=1.48$ and no-wind tunnel co-flow, $M_{W T}=0$. Both 8 and $31.5 \mathrm{kHz}$ octave presented.

Figures 19 and 20 show the two octave bands for the same CV jet conditions except that the wind tunnel flow is present. The subsonic cases seem to have few differences except they have somewhat decreased levels. For the supersonic case, the images of the shock cell noise emissions for the two bands show increased levels, but are somewhat more blurred than the no wind tunnel flow case. (Note the change of window size scale in Fig. 20.) 


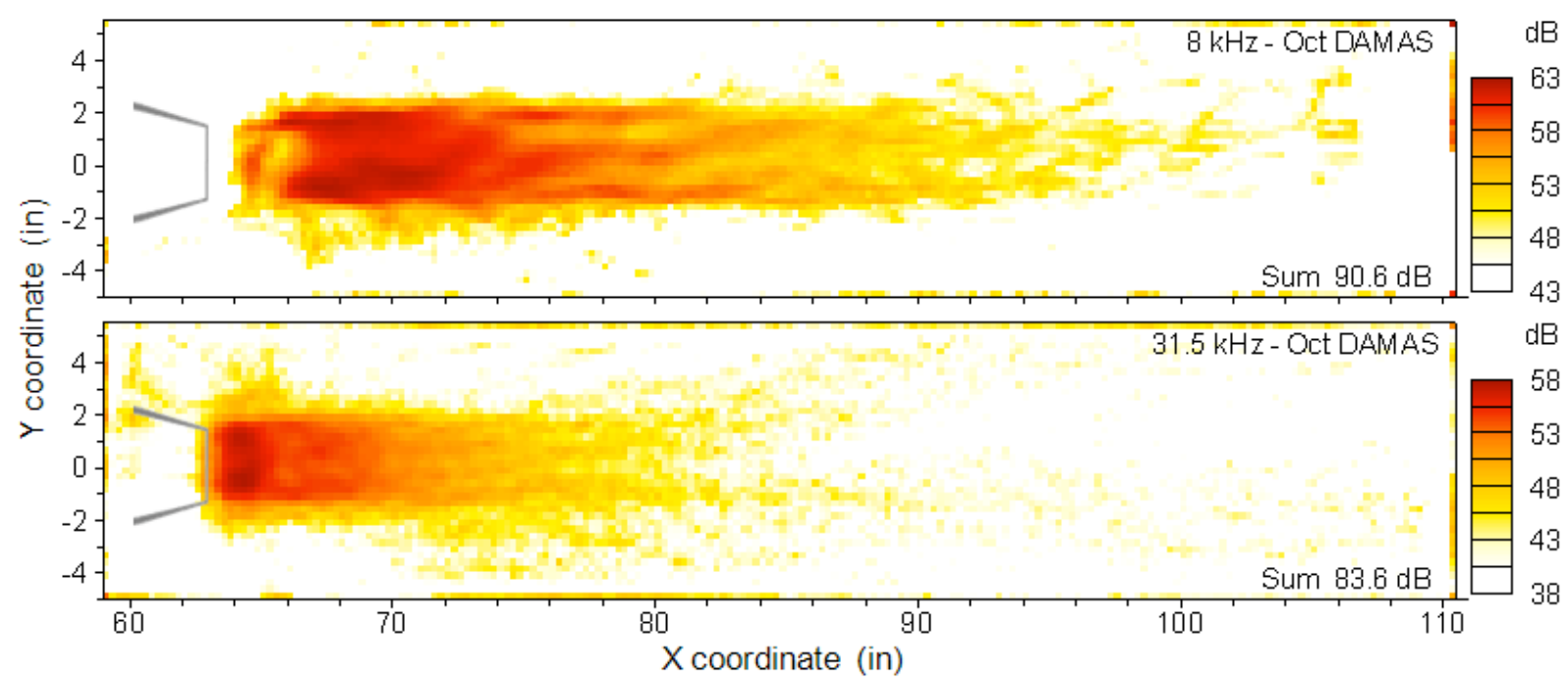

Figure 19. Single CV nozzle for subsonic cold jet at $M_{J}=0.8$ with wind tunnel co-flow, $M_{W T}=0.1$.

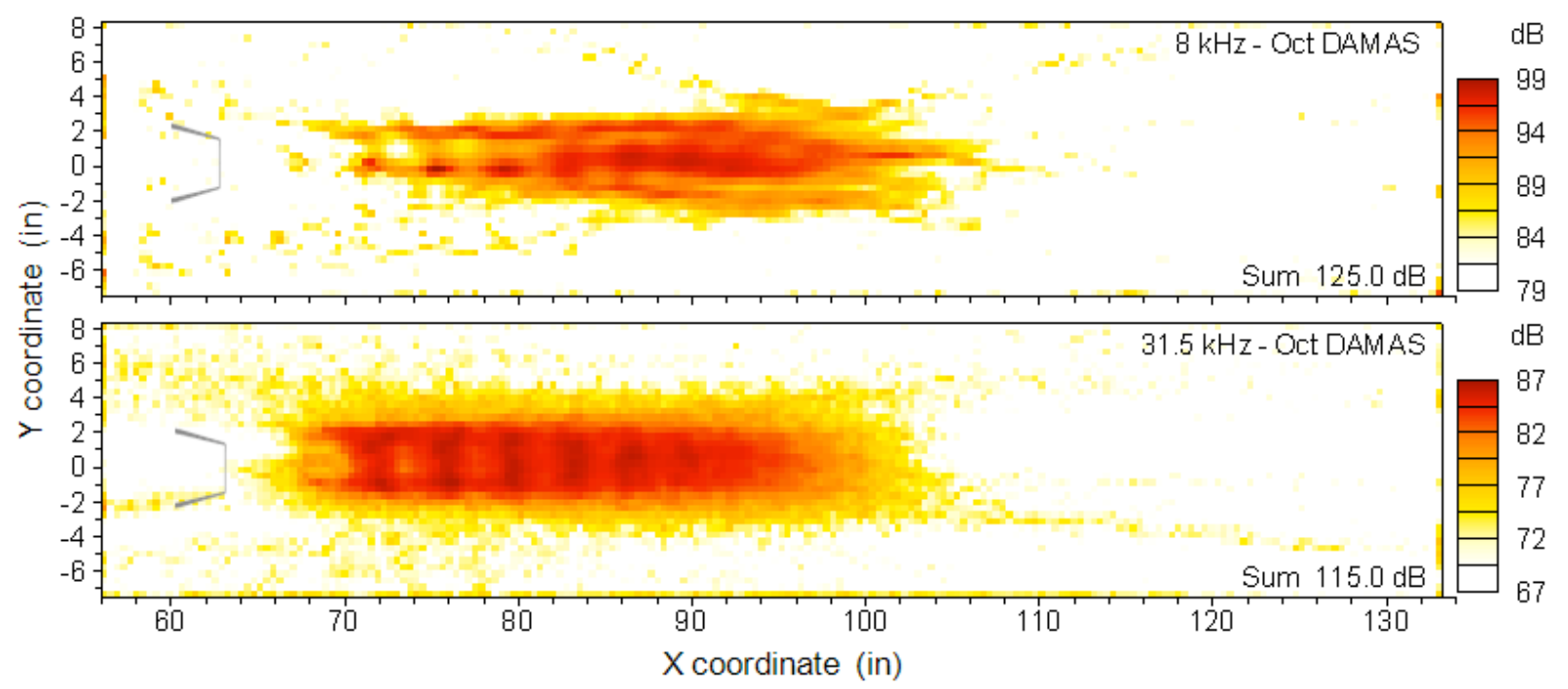

Figure 20. Single CV nozzle for supersonic cold jet at $M_{J}=1.48$ with wind tunnel co-flow, $M_{W T}=0.1$.

The convergent-divergent (CD) nozzle, Fig. 2 (b), has results shown in Fig. 21 for the same exit subsonic Mach number, $M_{J}=0.8$, and for the same frequency bands as Fig. 17 for the CV nozzle. Here, there is no wind tunnel flow, $M_{W T}=0$, the core NPR $=1.52$, the $T_{J}=100^{\circ} \mathrm{F}$, and $T_{\text {test }}=59.8^{\circ} \mathrm{F}$. For this subsonic over-expanded jet condition, the dominant noise present should be mixing noise. The total noise levels for the two bands are increased with this CD nozzle, compared to the CV nozzle, but the noise for both frequencies are concentrated about one D downstream of the nozzle exit. Noise emission continues for a number of D's downstream but the local levels are about $10 \mathrm{~dB}$ down. Figure 22 shows results for the CD nozzle with wind tunnel co-flow, $M_{W T}=0.1$. For the two frequency bands, levels decrease somewhat and appear to emit noise closer to the nozzle. Figure 23 shows the same bands for a matched supersonic CD case, $M_{J}=1.48$, with the same wind tunnel flow, $M_{W T}=0.1$. This CD nozzle is seen to be some $10 \mathrm{~dB}$ lower in level compared to the CV nozzle. The image shows evidence of broadband shock cell noise emission areas, but this is not as clear as that for the $\mathrm{CV}$ noise.

There are several single-nozzle hot flow cases examined. Figure 24 shows DAMAS results for the CV nozzle of Fig. 2 (a) for $M_{J}=0.8$, with wind tunnel flow of $M_{W T}=0.1$, a core NPS $=1.52, T_{J}=1000^{\circ} \mathrm{F}$, and $T_{\text {test }}=67.8^{\circ} \mathrm{F}$. Figure 23 shows results with similarity to Fig. 19 for cold tests except these levels are higher by about $10 \mathrm{~dB}$. Of course, this level reflects a large increase in jet speed at this Mach number. The $8 \mathrm{kHz}$ band shows signs of coherence with image streaking and stacking of high levels on the window boundary. The $31.5 \mathrm{kHz}$ band shows no 
evidence of this. A similar concentration of higher noise level occurs at the top and bottom edges of the images near the nozzle.

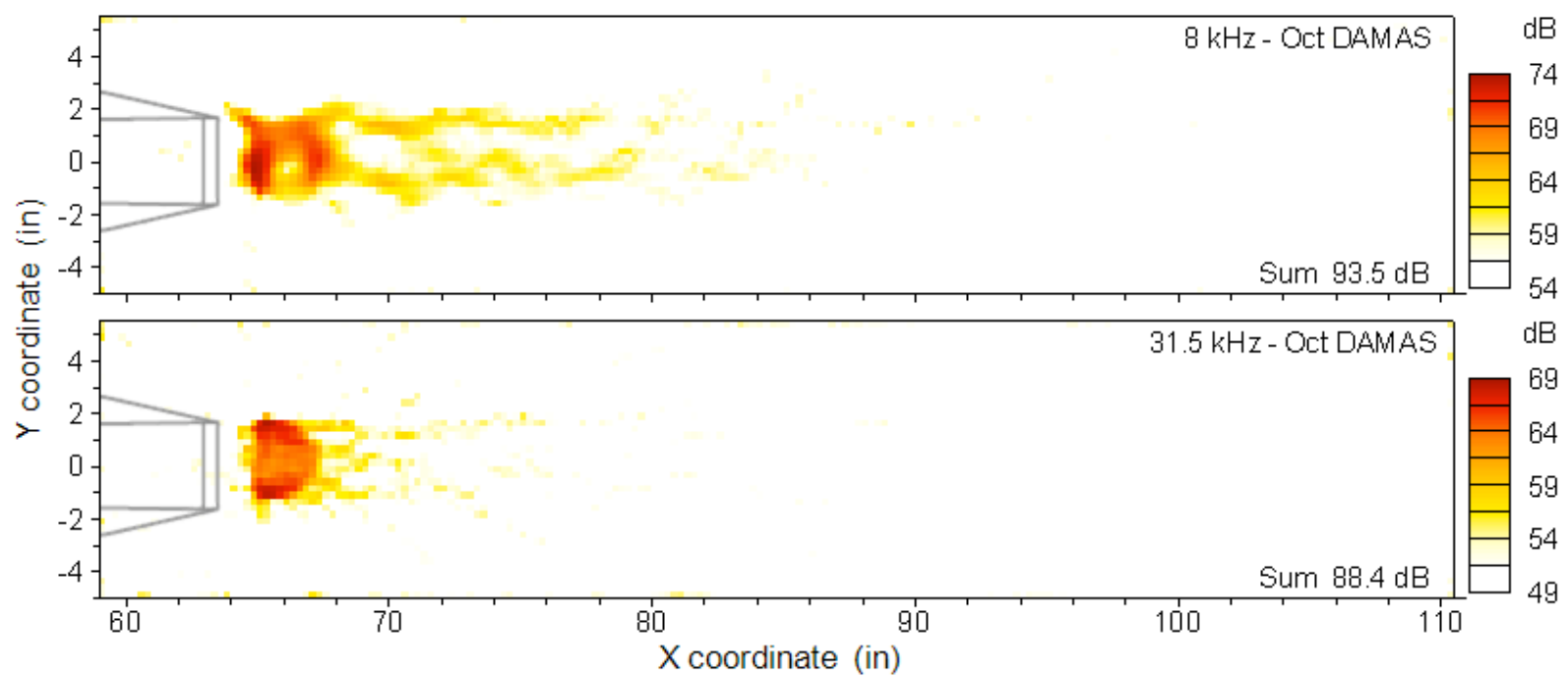

Figure 21. Single CD nozzle for subsonic cold jet at $M_{J}=0.8$ with no wind tunnel co-flow, $M_{W T}=0$.

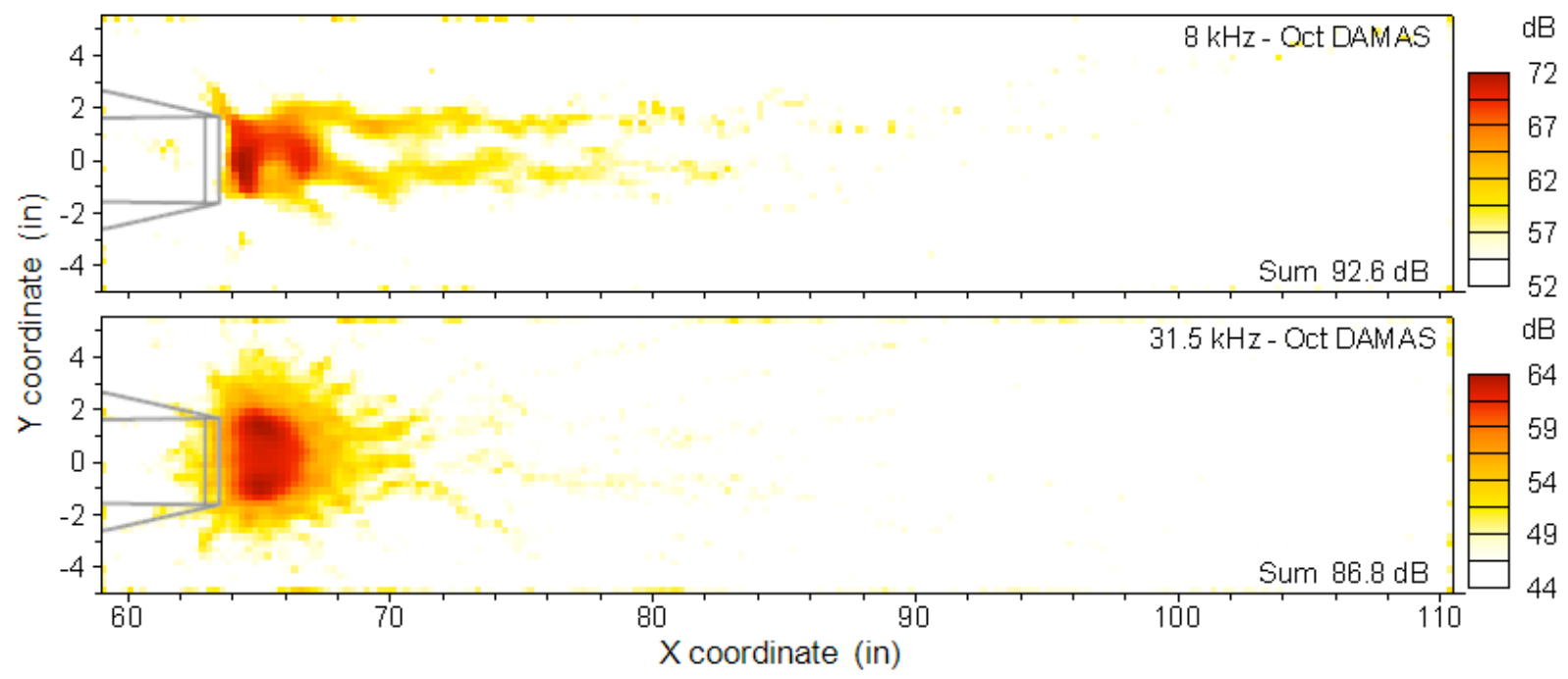

Figure 22. Single CD nozzle for subsonic cold jet at $M_{J}=0.8$ with wind tunnel co-flow, $M_{W T}=0.1$.

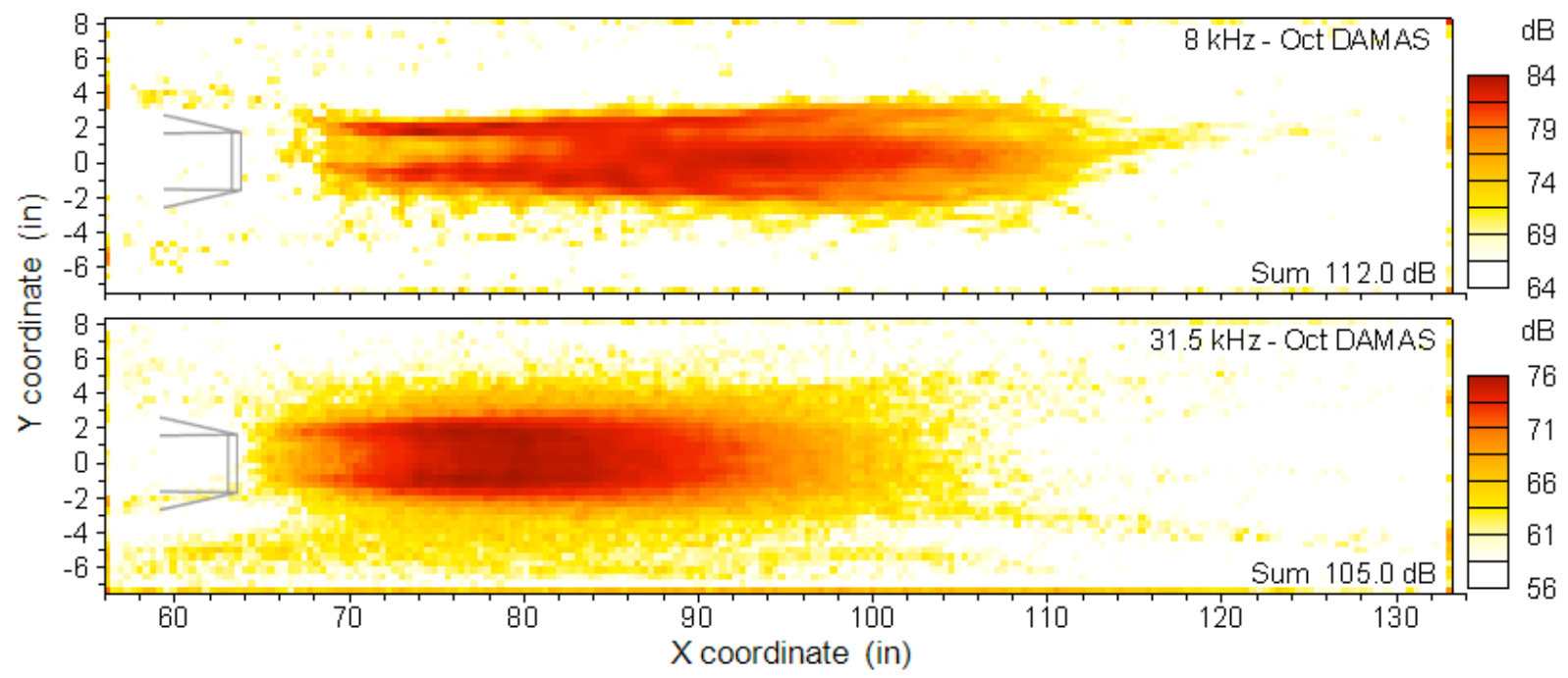

Figure 23. Single CD nozzle for supersonic cold jet at $M_{J}=1.48$ with wind tunnel co-flow, $M_{W T}=0.1$. 
Another supersonic single-nozzle hot flow case is shown in Fig. 25 for the CD nozzle of Fig. 2 (b), for $M_{J}=$ 1.45 , the wind tunnel flow at $M_{W T}=0.1$, a core NPR $=3.42$, a $T_{J}=1000^{\circ} \mathrm{F}$, and a $T_{\text {test }}=65.5^{\circ} \mathrm{F}$. One sees by comparing this with a similar Mach number of $M_{J}=1.48$, in Fig. 23, that levels are 4-5 dB higher (with the increase in jet speed at this temperature) and jet plume noise production geometry changes. The $8 \mathrm{kHz}$ band shows strong signs of coherence with substantial streaking and window boundary noise levels. The $31.5 \mathrm{kHz}$ band show perhaps some signs of this.

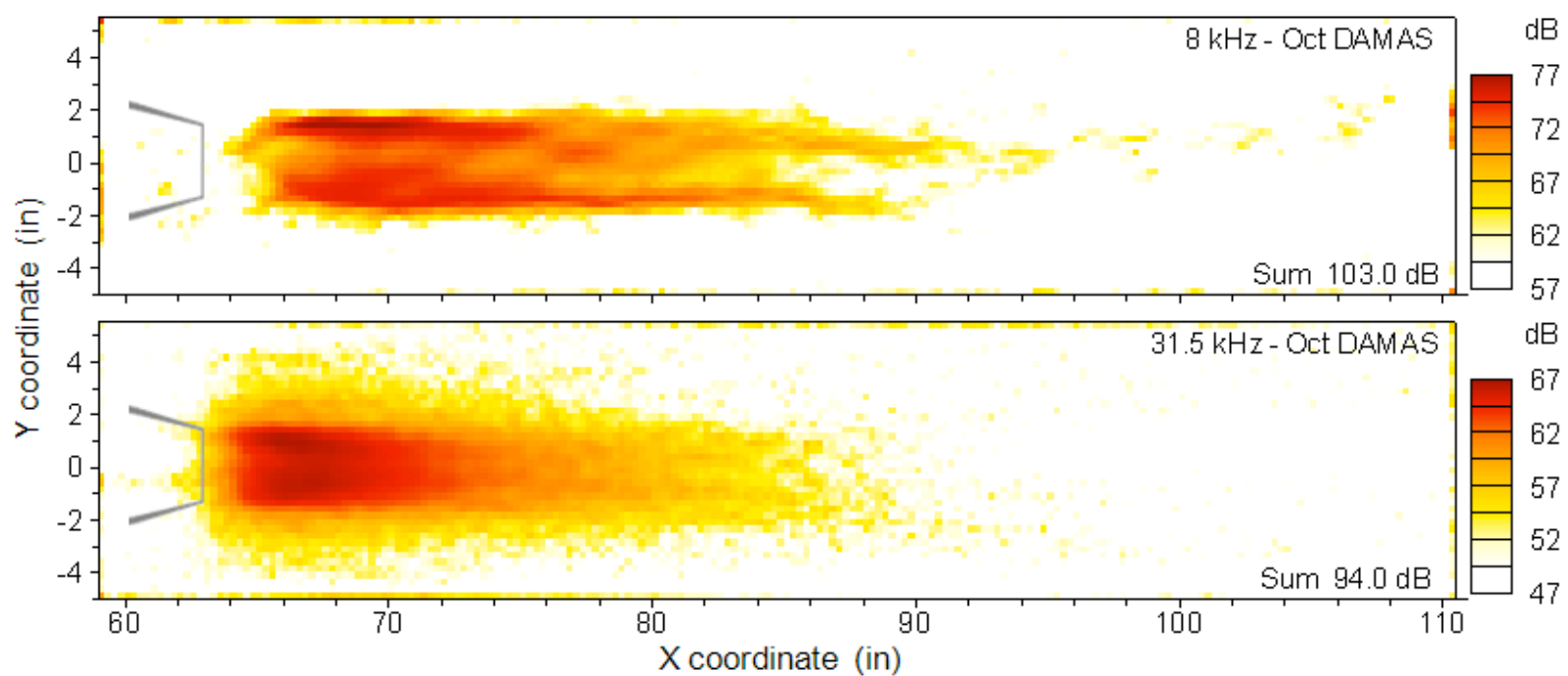

Figure 24. Single CV nozzle for subsonic hot jet at $M_{J}=0.8$ with wind tunnel co-flow, $M_{W T}=0.1$.

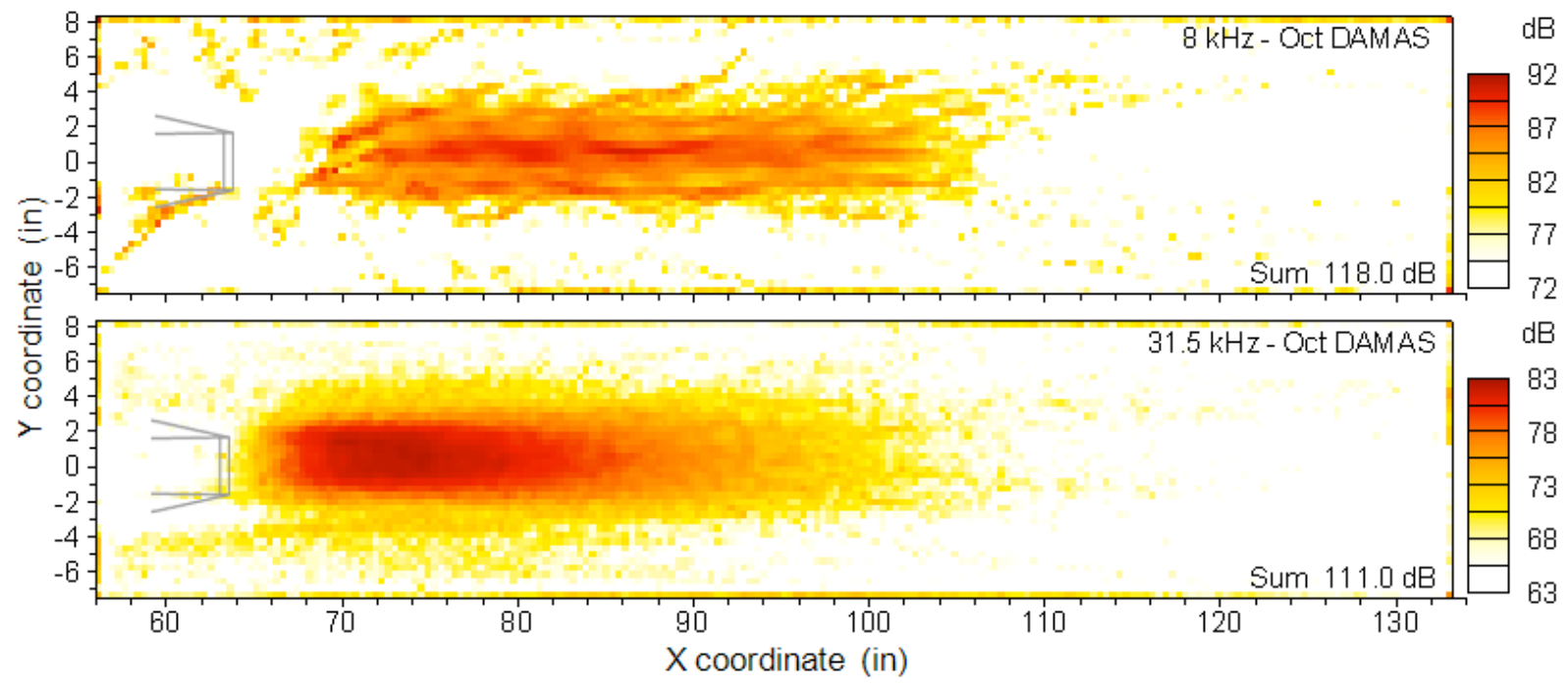

Figure 25. Single CD nozzle for supersonic hot jet at $M_{J}=1.45$ with wind tunnel co-flow, $M_{W T}=0.1$.

Dual-Flow Jet Nozzle Configurations: BPR 5 Chevron Study. In this section, several dual-flow nozzle configurations are examined for a limited range of conditions. The first are results of a series of chevron fan and core nozzles employed in the Thomas and Kinzie ${ }^{10}$ study. The $90^{\circ}$ observation angle results for the present study are given in Figs. 26, 27, 28, and 29, for the same two full-octave frequency bands used for the single-jet cases of the last section. The configurations correspond to Figs. 2 (d), (e), (f), and (g). The orientations of the chevrons matched that shown in Fig. 3. Test conditions for all cases were for a core NPR $=1.56$, core TTR $=2.66$, fan NPR $=1.75$, and fan TTR $=1.16$. There is a wind tunnel flow at $M_{W T}=0.1$ and the ambient temperature, $T_{\text {test }} \approx 77.0^{\circ} \mathrm{F}$. For the Baseline configuration in Fig. 26, it is apparent that there is substantial coherence over the $8 \mathrm{kHz}$ octave frequency range. The image appears fractured and has a stacking of some strong levels around the edges of the scanning plane. The overall level should be correct at $102 \mathrm{~dB}$. The image for the $31.5 \mathrm{kHz}$ band is quite different. The image is 
balanced and has higher levels along the nozzle lip lines (top and bottom) downstream of the exit. The flow is seen to converge downstream of the external plug, and consequently expands. The overall level is $93.1 \mathrm{~dB}$. Figure 27 shows that the core chevrons have little effect on the radiated noise at this $90^{\circ}$ array position. Figure 28 shows that the fan chevrons have a strong influence on the location of the noise sources - after several fan nozzle diameters there is little noise emitted. However, the total noise is seen to increase in level by three to five dB. A similar source map is shown in Fig. 29, when both the fan and core have chevrons. Again the core chevrons show little noise reduction benefit at this $90^{\circ}$ observation position.

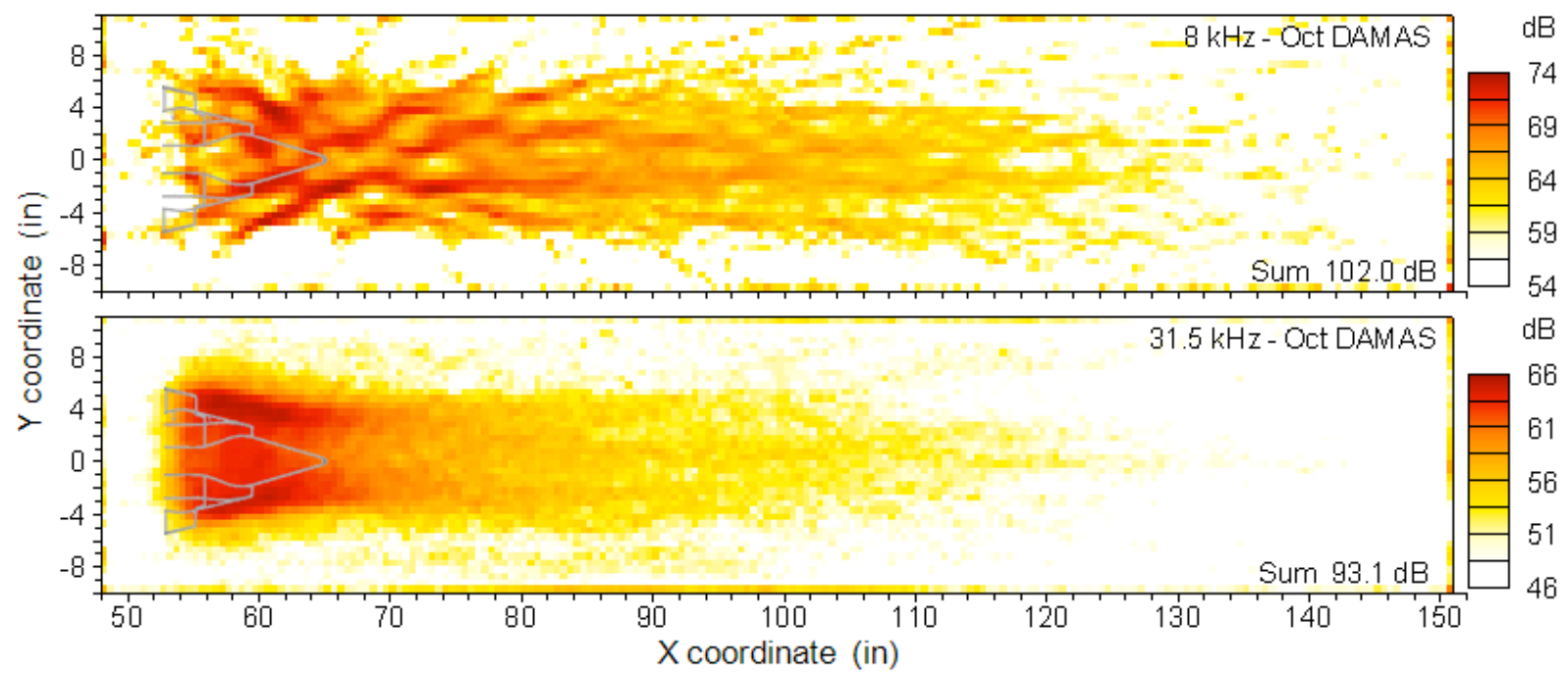

Figure 26. BPR5 dual-flow nozzle, no Chevrons, BASELINE (Chevron Study). Wind tunnel flow, $M_{W T}=0.1$.

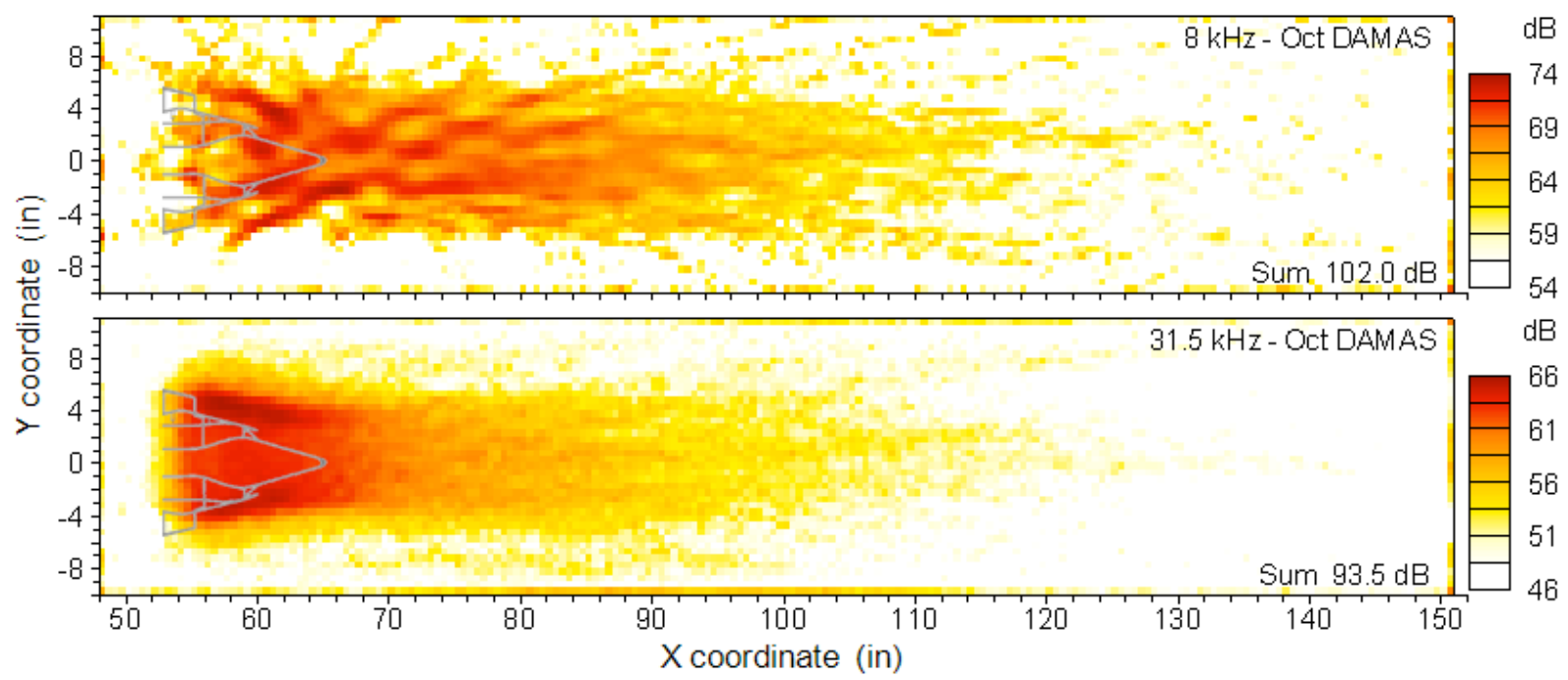

Figure 27. BPR5 dual-flow nozzle, CORE Chevrons Only, (Chevron Study). Wind tunnel flow, $M_{W T}=0.1$.

Dual-Flow Jet Nozzle Configurations: BPR 11 Core/Fan Study. For this section, results are given for an internal-plug convergent nozzle configuration with a convergent fan nozzle, shown in Fig.2 (c). The core nozzle is the same as that of Fig. 2 (a) and the fan nozzle is the same as the baseline nozzle of Fig. 2 (d). The nozzle flows are cold at $T_{\text {Fan }}=T_{\text {Core }}=100^{\circ} \mathrm{F}$. The test temperature is $T_{\text {test }} \approx 79^{\circ} \mathrm{F}$. There is no wind tunnel flow, $M_{W T}=0$. Figure 30 shows the DAMAS results for the core jet operating alone, such that $M_{\text {CoreJ }}=1.0$ and $M_{\text {FanJ }}=0$. (Core NPR $=1.89$ and the Fan NPR $=1.0$.) This sonic Mach number can be compared to the $M_{\text {CoreJ }}=0.8$ result in Fig. 13 . It is seen that for both the 8 and $31.5 \mathrm{kHz}$ octave band frequencies that noise emission is concentrated more downstream and is of higher level. Figure 31 shows the results with the core-jet turned off and the fan-jet operating alone, with $M_{\text {CoreJ }}=0$ and $M_{\text {FanJ }}=0.71$. (Core NPR $=1.0$ and the Fan NPR = 1.4.) Note the change of scale. The fan noise production is seen to contract downstream, as it passes the core nozzle exit - then expand several fan diameters downstream. Next, Fig. 32 shows the results for both the core and fan-jet operating at $M_{\text {CoreJ }}=1.0$ and 


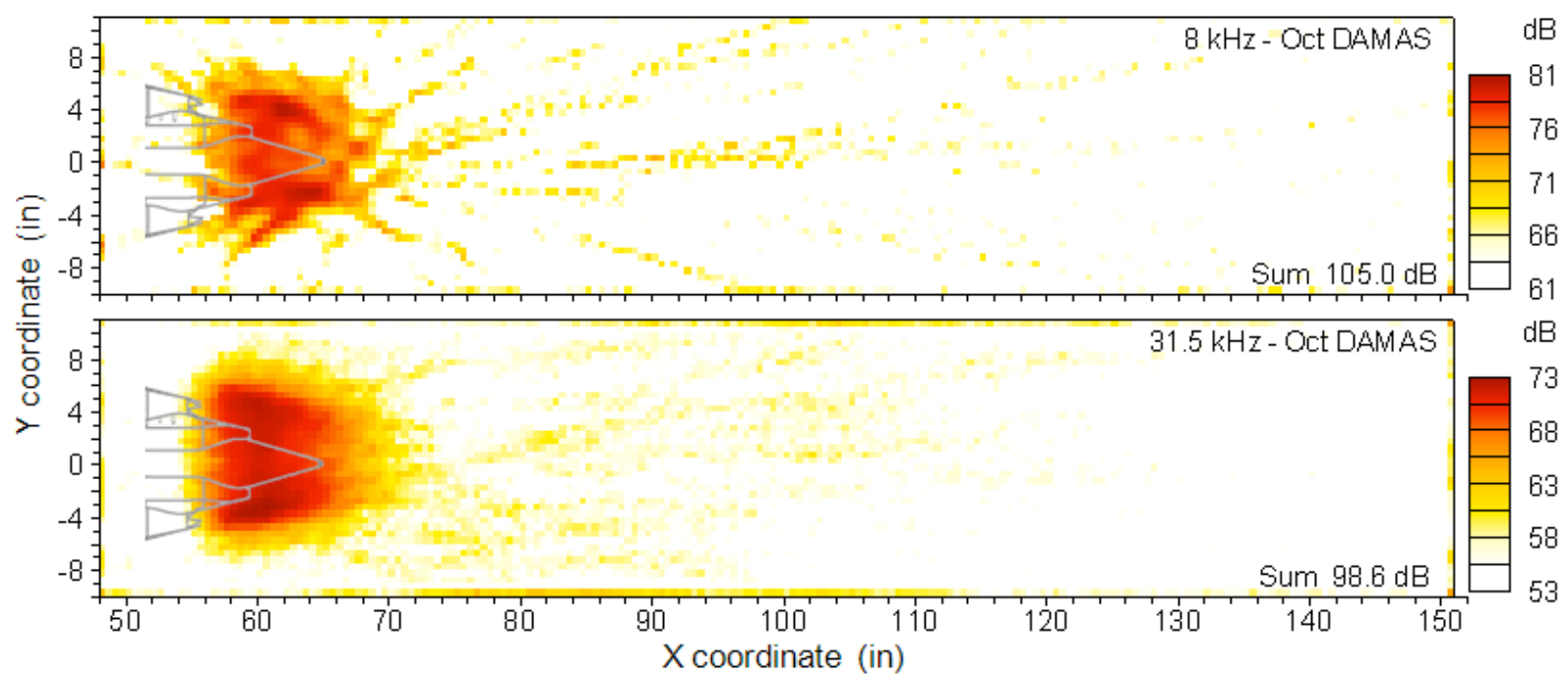

Figure 28. BPR5 dual-flow nozzle, FAN Chevrons Only, (Chevron Study). Wind tunnel flow, $M_{W T}=0.1$.

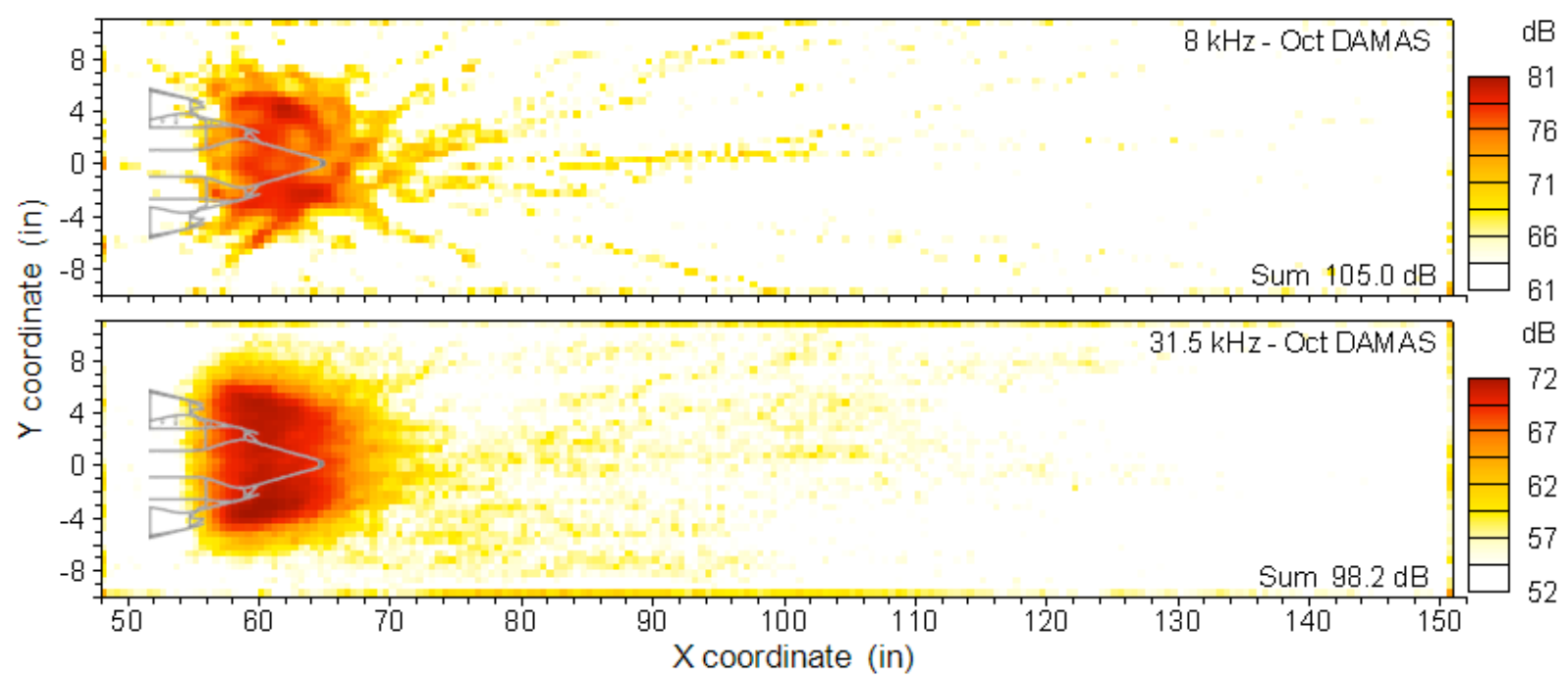

Figure 29. BPR5 dual-flow nozzle, Both CORE and FAN Chevrons, (Chevron Study). Wind tunnel flow, $M_{W T}=0.1$.

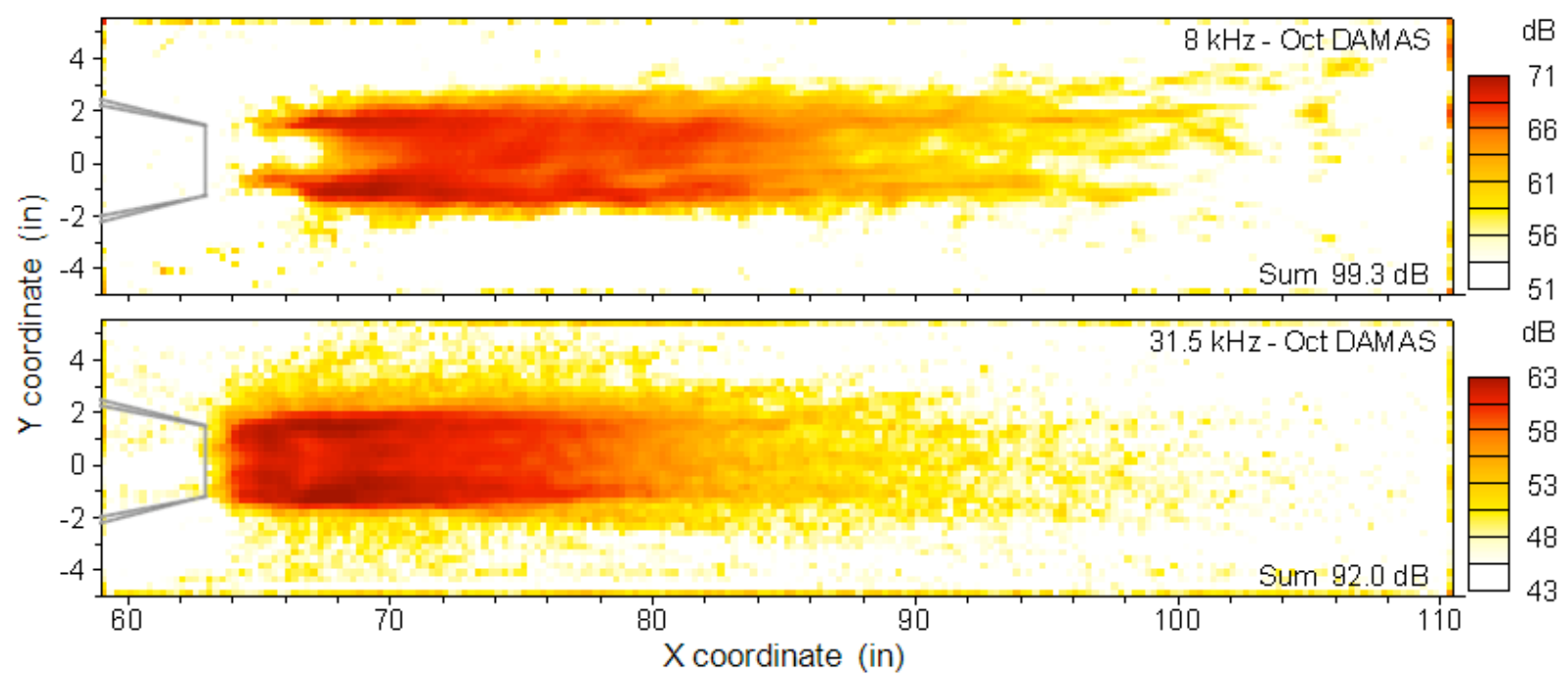

Figure 30. BPR11 dual-flow nozzle, Core jet only operating, $M_{\text {CoreJ }}=1.0$ and $M_{\text {FanJ }}=\mathbf{0}$. No wind tunnel flow, $M_{W T}=0$. (Note difference in this window size compared to the following BPR11 nozzle cases.) 


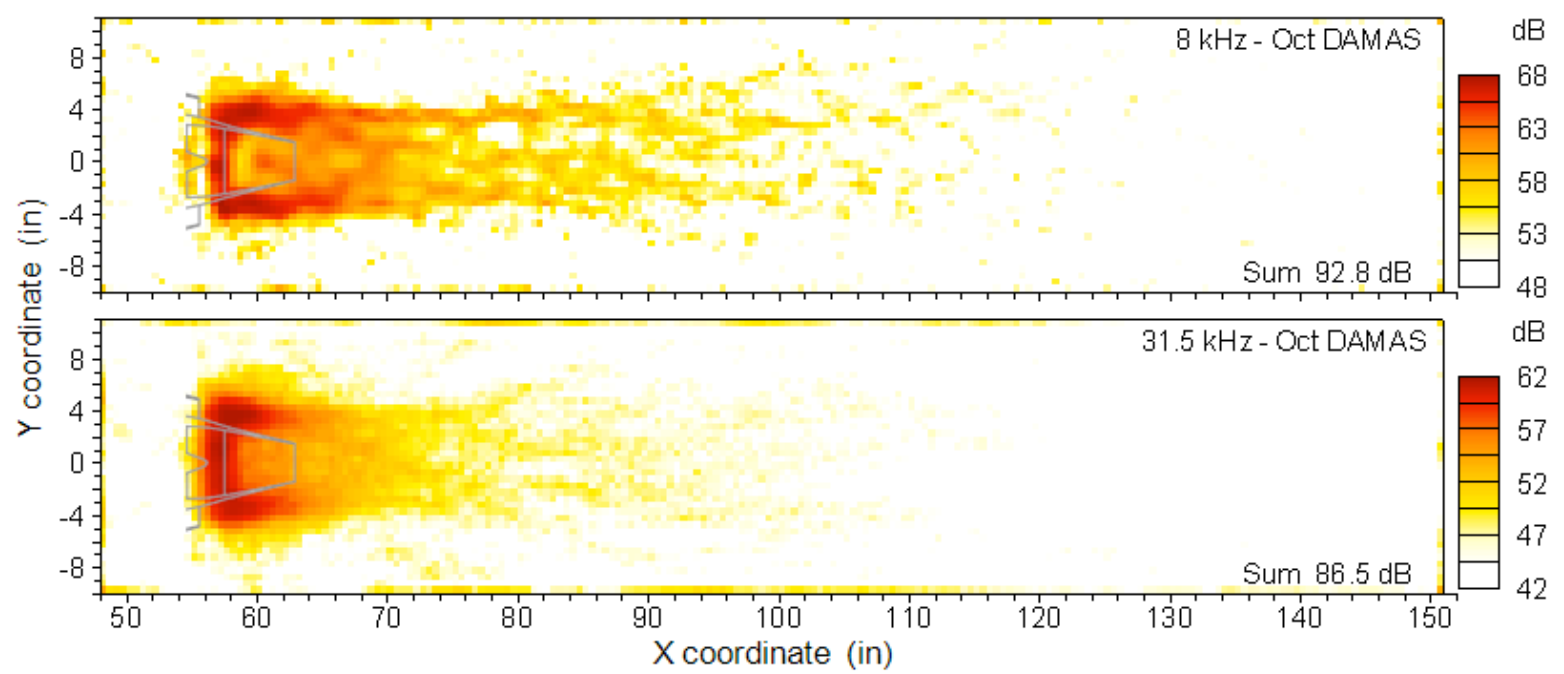

Figure 31. BPR11 dual-flow nozzle, Fan jet only operating, $M_{\text {CoreJ }}=\mathbf{0}$ and $M_{\text {FanJ }}=\mathbf{0 . 7 1}$. No wind tunnel flow.

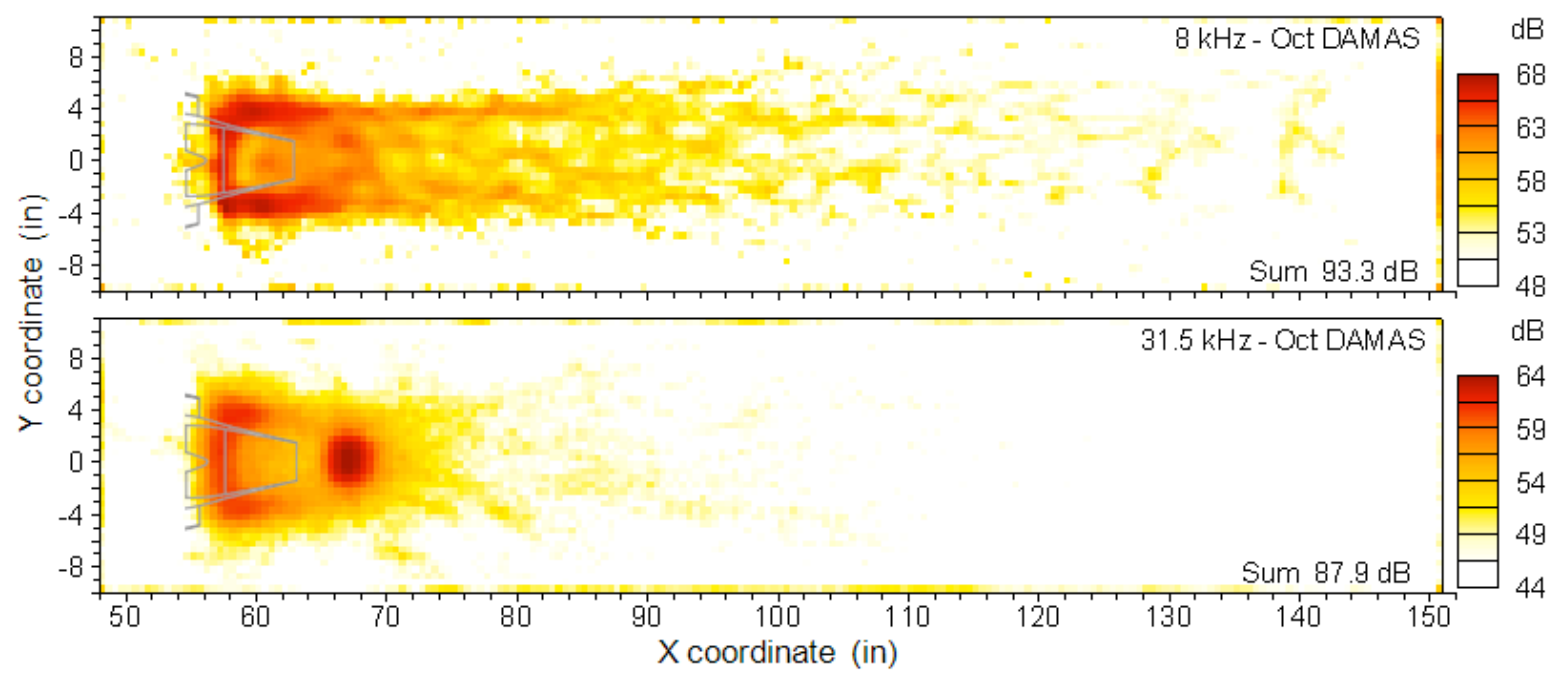

Figure 32. BPR11 dual-flow nozzle, Fan and Core Jet operating, $M_{\text {CoreJ }}=\mathbf{1 . 0}$ and $M_{\text {FanJ }}=\mathbf{0 . 7 1}$. No wind tunnel flow, $M_{W T}=\mathbf{0}$.

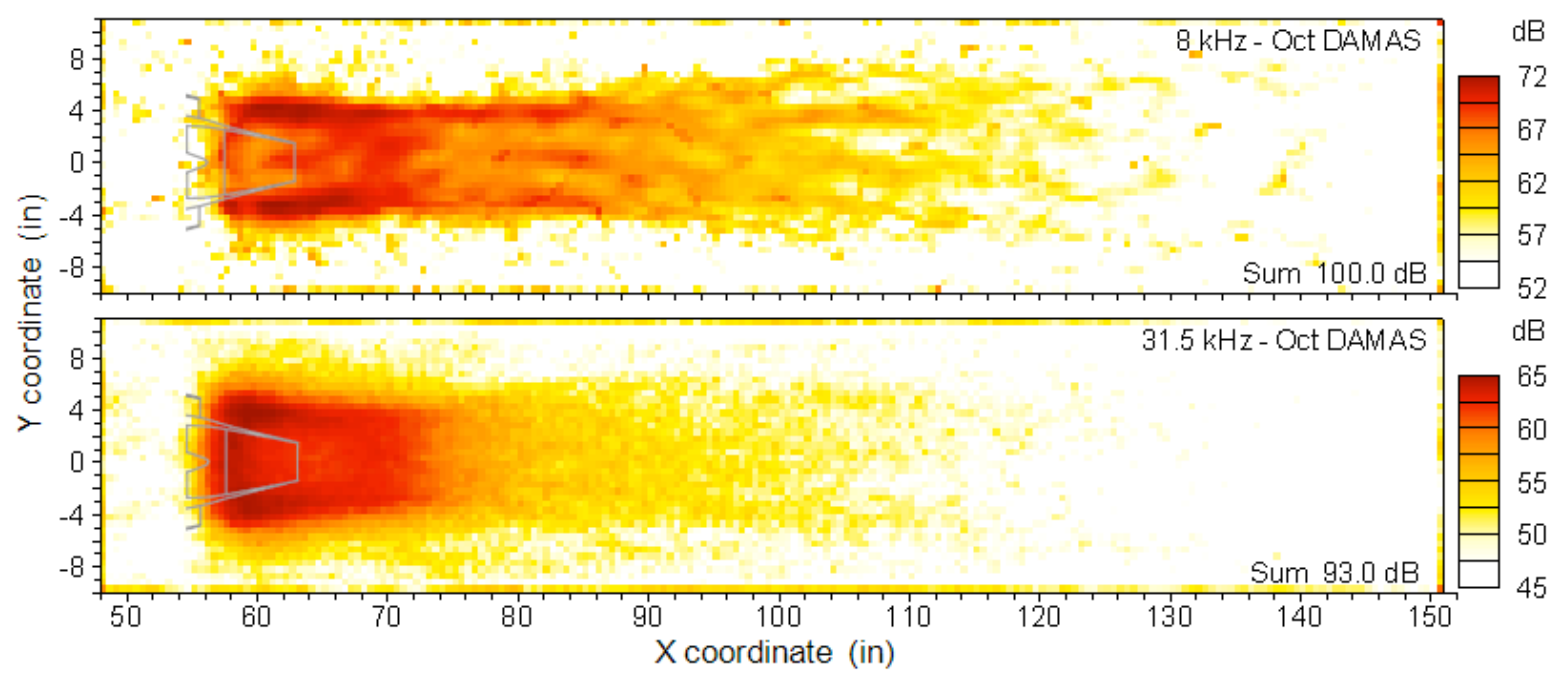

Figure 33. BPR11 dual-flow nozzle, Fan jet only operating, $M_{C o r e J}=0$ and $M_{F a n J}=0.93$. No wind tunnel flow, $M_{W T}=\mathbf{0}$. 


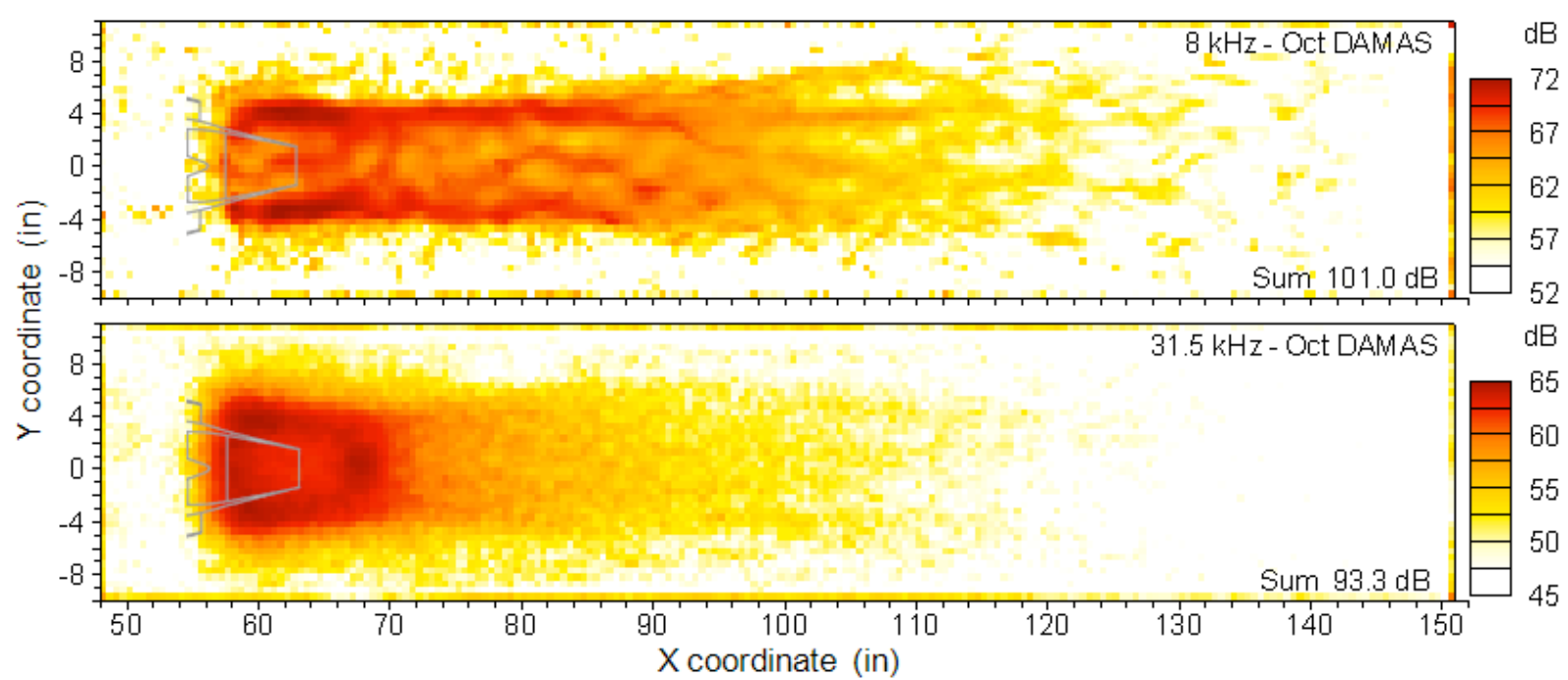

Figure 34. BPR11 dual-flow nozzle, Both Fan and core jet operating, $M_{\text {CoreJ }}=1.0$ and $M_{\text {FanJ }}=0.93$. No wind tunnel flow, $M_{W T}=\mathbf{0}$.

$M_{\text {FanJ }}=0.71$. (Core NPR $=1.89$ and the Fan NPR $=1.4$.) A contribution from the core is seen near coordinate $X=67$ ". The levels are only about one-half dB higher than without the core-jet. Also, it is 4 to $6 \mathrm{~dB}$ lower than the core-jet operating alone. Figure 33 shows the results for the fan-jet operating alone, but at a higher Mach number than that of Fig. 31 . Here the $M_{\text {CoreJ }}=0$ and the $M_{\text {FanJ }}=0.93$. (Core NPR $=1.0$ and the Fan NPR = 1.75.) Note the approximate $5 \mathrm{~dB}$ increase at this speed. Figure 34 shows the results for both the core and fan-jet operating, at $M_{\text {CoreJ }}=1.0$ and $M_{\text {FanJ }}=0.93$. (Core NPR $=1.89$ and the Fan NPR $=1.75$.) Here, only about a dB or less increase in level from the fan alone case is seen.

\section{CONCLUSIONS}

A microphone array study is reported of subsonic and supersonic jet noise. The microphone array (named JEDA) acquired data at $90^{\circ}, 115^{\circ}, 135^{\circ}$, and $150^{\circ}$ to the jet axis for all nozzle configurations, engine cycles, and wind tunnel speeds tested. The jet noise cases included cold and hot, single flow convergent and convergentdivergent nozzles at differing subsonic and supersonic flow conditions. This was done with and without wind tunnel co-flow. Also dual-stream nozzles with hot core flow of different bypass ratios (BPR) were studied to determine the effect of the use of chevrons on the core and fan nozzles. A separate BPR circular nozzle configuration was used to examine and separate contributions from the fan and core nozzles for several different engine cycles.

A method is presented to calibrate the array's "focus" for array positions with respect to the noise survey region. The method accounts for any error in the microphone-to-grid point steering vectors, which are based on Amiet shear layer corrections for open wind tunnels. The method depends on a "conditioning" of the array crossspectral matrix prior to beamforming and DAMAS calculations. The method results in sharper and properpositioned images.

This paper demonstrates the usefulness of the deconvolution algorithm DAMAS to process jet noise data. Cases examined are only for the array at its $90^{\circ}$ position normal to the flow direction. Jet noise mapping is shown for frequencies above any screech dominance. The frequency ranges emphasized are the $8 \mathrm{kHz}$ and $31.5 \mathrm{kHz}$ octave bands, which with a scale factor of 10 would correspond to $800 \mathrm{~Hz}$ and $3.15 \mathrm{kHz}$ full scale. DAMAS was found to allow quantitative determination of noise source locations in the jet plume. DAMAS assumes distributions of statistically independent sources over the grid point window. Noise mapping gives indications when a source distribution can or cannot be constructed by being cast in such terms. When independent sources can be assumed, the images tend to be clear, smooth, and distinct. When not, the images become fractured and strong source strength is attributed to the grid window edges. This can also happen when the window is not properly sized. There are a number of cases shown that indicate that coherence is strong enough that the assumptions of DAMAS are not valid. 
Here the use of DAMAS-C (a coherent source version of DAMAS) or another deconvolution method that deals with coherence may be considered. This is beyond the scope of the present paper. Still, DAMAS gives the total noise correctly and indicates where coherence is located.

\section{ACKNOWLEGMENTS}

The author would like to thank Dr. Brenda Henderson, Mr. Henry Haskin, Mr. Thomas Norum, and Mr. John Swartzbaugh of the Jet Noise Laboratory. Particularly, Dr. Henderson (now at NASA-GRC) encouraged the test program and provided excellent management of resources. Mr. Haskin provided outstanding design and other support.

\section{REFERENCES}

1. B.J. Tester and S.A.L. Glegg, “A Review of Engine Noise Source Diagnostic Methods for Static Engine Tests, with Phased Array and Polar Correlation Techniques”, AIAA-2008-2854.

2. D. Papamoschou and A. Dadvar, “Localization of Multiple Types of Jet Noise Sources”, AIAA-2006-2644.

3. T.F. Brooks and W.M. Humphreys, Jr., "A Deconvolution Approach for the Mapping of Acoustic Sources (DAMAS) Determined from Phased Microphone Arrays", Journal of Sound and Vibration, Vol. 294, pp. 856-879, 2006.

4. R.P. Dougherty and G.G. Podboy, "Improved Phased Array Imaging of a Model Jet”, AIAA 2009-3186.

5. P. Sijtsma, “CLEAN Based on Spatial Source Coherence”, AIAA-2007-3436.

6. J.A. Hogbom, “Aperture Synthesis with a Non-Regular Distribution of Interferometer Baselines”, Astron. Astrophys. Suppl., No. 15, 1974, pp. 417-426.

7. R.P. Dougherty, “Extensions of DAMAS and Benefits and Limitations of Deconvolution in Beamforming”, AIAA- $2005-2961$.

8. T.F. Brooks and W.M. Humphreys, "Extension of DAMAS Phased Array Processing for Spatial Coherence Determination (DAMAS-C)”, AIAA-2006-2654.

9. T.D. Norum, “Reductions in Multi-component Jet Noise by Water Injection”, AIAA-2004-2976.

10. R.H. Thomas and K.W. Kinzie, “Jet-Pylon Interaction of High Bypass Ratio Separate Flow Nozzle Configurations", AIAA2004-2827.

11. R.K. Amiet, R. K., “Refraction of Sound by a Shear Layer,” Journal of Sound and Vibration, vol. 58, no. 3, pp. 467-482, 1978.

12. T.F. Brooks and W.M. Humphreys, Jr., "Three-Dimensional Application of DAMAS Methodology for Aeroacoustic Noise Source Definition”, AIAA-2005-2960.

13. J.M. Mendoza, T.F. Brooks, and W. M. Humphreys, Jr., "An Aeroacoustic Study of a Leading Edge Slat Configuration," International Journal of Aeroacoustics, vol. 1, pp. 241-274, 2009.

14. W.M. Humphreys, Jr., and T.F. Brooks, "Noise Spectra and Directivity for a Scale-model Landing Gear", International Journal of Aeroacoustics, vol. 8, pp. 409-444, 2002.

15. W. Humphreys, T. Brooks, W. Hunter, and K. Meadows: "Design and Use of Microphone Directional Arrays for Aeroacoustics Measurements", AIAA paper 98-0471. 